\title{
A three-dimensional coupled Nitsche and level set method for electrohydrodynamic potential flows in moving domains
}

\author{
A. Johansson* \\ Center for Biomedical Computing, Simula Research Laboratory, Norway \\ M. Garzon* \\ Department of Applied Mathematics, University of Oviedo, Spain \\ J. A. Sethian* \\ Mathematics Department, Lawrence Berkeley National Laboratory, \\ Department of Mathematics, University of California, Berkeley, United States
}

\begin{abstract}
In this paper we present a new algorithm for computing three-dimensional electrohydrodynamic flow in moving domains which can undergo topological changes. We consider a non-viscous, irrotational, perfect conducting fluid and introduce a way to model the electrically charged flow with an embedded potential approach. To numerically solve the resulting system, we combine a level set method to track both the free boundary and the surface velocity potential with a Nitsche finite element method for solving the Laplace equations. This results in an algorithmic framework that does not require body-conforming meshes, works in three dimensions, and seamlessly tracks topological change. Assembling this coupled system requires care: while convergence and stability properties of Nitsche's methods have been well studied for static problems, they have rarely been considered for moving domains or for obtaining the gradients of the solution on the embedded boundary. We therefore investigate the performance of the symmetric and non-symmetric Nitsche formulations, as well as two different stabilization techniques. The global algorithm and in particular the coupling between the Nitsche solver and the level set method are also analyzed in detail. Finally we present numerical results for several time-dependent problems, each one designed to achieve a specific objective: (a) The oscillation of a perturbed sphere, which is used for convergence studies and the examination of the Nitsche methods; (b) The break-up of a two lobe droplet with axial symmetry, which tests the capability of the algorithm to go past flow singularities such as topological changes and preservation of an axi-symmetric flow, and compares results to previous axi-symmetric calculations; (c) The electrohydrodynamical deformation of a thin film and subsequent jet ejection, which will account for the presence of electrical forces in a non axi-symmetric geometry.
\end{abstract}

Keywords: Nitsche finite element method, Level set method, Potential flow, Electrostatic driven flows

\section{Motivation and overview}

Understanding the mechanism of fluid break-up is a key element in various scientific and industrial applications, such as wave breaking, fluid-structures interaction, and droplet and bubble pinch-off. The

*Corresponding author. Address: Center for Biomedical Computing, Simula Research Laboratory, P.O. Box 134 , 1325 Lysaker, Norway. Tel: +4740451301; fax +4767828201

Email addresses: august@simula.no (A. Johansson), maria.garzon.martin@gmail.com (M. Garzon), sethian@math.berkeley.edu (J. A. Sethian) 
inclusion of electrified jets (and drops), which occurs in such areas as polymer melts [1, storm cloud formation [2], commercial ink jet printing [3], and particle removal from drop surfaces [4] adds additional complications. As one example, Marginean et al. [5] have experimentally studied the complicated nature of electrospraying and the diversity of bursting modes that arise. For opposite charged droplets, recent experiments [6] have revealed the coalescence or bouncing regimes between droplets depending upon the intensity of the applied electric field.

Beginning with the pioneering work of Lord Rayleigh 7 and G. I. Taylor $[8,9$, a vast literature has been devoted to understanding fluid breakup and motion induced by electric fields. The mathematical models and numerical algorithms to tackle these phenomena are diverse and rely on different assumptions. Although the general Navier-Stokes equations is the appropriate to model fluids, its complexity and computational cost in three dimensions have motivated the use of various simplifying assumptions. For low viscous fluids like water, the motion can be considered inviscid and irrotational up to length scales of few nanometers. Nonetheless, singular flow events may occur, such as drop breaking or merging, and the subsequent undamped capillary surface waves generated remain a challenge for most of the existing fluid dynamic algorithms.

One method for potential flow computation in moving and breaking domains uses the level set embedding techniques to establish an Eulerian formulation of the classical Lagrangian equations [10, 11]. The advantage of this approach is that it seamlessly allows topologically breakup of droplets. It has proven to be a robust method in simulating various physical situations, such as wave overturning and breaking, see [10; the TaylorRayleigh instability of a fluid jet, 11; droplet and bubble evolution in a two fluid system, 12, 13; and more recently electrical droplet deformation, [14.

In this approach, the fluid interface and accompanying fluid velocity potential are embedded in higher dimensional functions, and hence allow the advantages of a level set approach. However, in the version presented in [14, the potential model was solved using a boundary integral formulation, which required explicit construction of the interface at each time step through a set of nodes. In the above droplet studies, an axisymmetric flow was assumed, making the construction of this interface straightforward.

In this paper, we introduce a natural extension of this work which takes full advantage of the level set approach, and incorporate a Laplace solver for the potential field that does not require an explicit, bodyconforming mesh. This allows us to track dynamic electrostatic effects on fully three-dimensional, non-mesh conforming electrically charged interfaces.

Our approach starts with the classical Nitsche finite element, as proposed in 1971 by Nitsche [15]. This method enforces Dirichlet boundary conditions using the variational form rather than the finite element spaces, as is usually the case. This makes it possible to develop finite element methods on meshes that do not have to conform to the computational domain. These methods can be of arbitrary order and can handle general boundary conditions. However, if the intersection of the domain and an element is zero, a breakdown of the finite element method may occur since the bilinear form is no longer be coercive. In this case numerical instabilities appear in the form of singular or very badly conditioned linear systems. For problems with moving boundaries, small volumes are likely to occur and this issue has to be addressed to obtain a robust method. Several methods that avoid these issues have been proposed, for example Burman's ghost penalty method [16] which will be tested here; additional methods include those introduced by Burman and Hansbo in a series of papers [17-19, as well as the approach by Johansson and Larson [20.

The goal of this paper is two-fold:

1. To analyze the family of Nitsche finite element methods and stabilization techniques when applied to static and dynamical problems in the context of the aforementioned applications.

2. To develop and implement a three-dimensional algorithm that couples the Nitsche finite element method with the level set method for the study of electrohydrodynamical problems; the algorithms are validated through a series of case studies.

The paper is organized as follows: In Section 2 we present the model equations for the interior fluid dynamics and exterior electric field using the classical Lagrangian formulation. The complete Eulerian formulation of the PDE system using the level set approach is also described briefly. In Section 3 an overall description of the Nitsche and level set coupling is introduced together with the basic algorithm. The Nitsche finite element methods and the stabilization techniques are covered in detail in Section 4 4 followed 


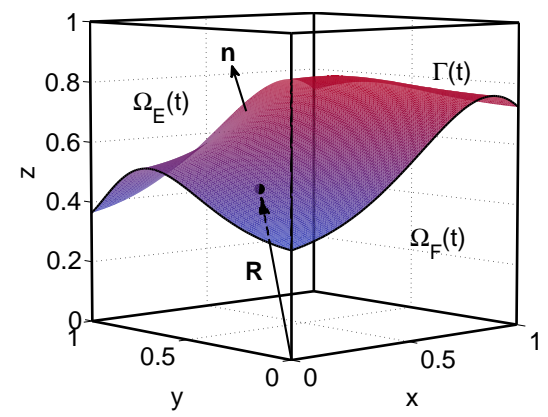

Figure 2.1: Schematic figure of the physical setting. $\Omega_{F}(t)$ is the fluid domain and $\Omega_{E}(t)$ is the exterior domain.

by a review of the level set method in Section 5 . Numerical tests to validate the method are presented in Section 6, and in Section 7 we show numerical results corresponding to an electrohydrodynamic example. Conclusions are given in Section 8

\section{The model equations}

To introduce the model equations we follow the presentation in [14 and consider two time-dependent regions occupied by different fluids and separated by a moving boundary. For the interested reader, a more detailed intrinsic derivation of the potential model equations can be found in 21 . We use the subscript $F$ for the liquid and $E$ for the exterior fluid. Let $\Omega_{F}(t), \Omega_{E}(t)$ be three-dimensional moving fluid domains, $\Gamma(t)$ a parameterization of the free surface between both domains at time $t \in[0, T]$ and $\mathbf{R}(\mathbf{s}, t), \mathbf{s}=\left(s_{1}, s_{2}\right)$ the position vector of a fluid particle on the moving front. See Fig. 2.1 for a 3D sketch of the physical domain.

We assume that the fluid in $\Omega_{E}(t)$ is at rest and $p_{a}$ is the constant reference pressure. The fluid occupying $\Omega_{F}(t)$ is considered incompressible, irrotational and inviscid, and thus the conservation laws of mass and momentum in $\Omega_{F}(t)$ lead to the classical potential Eulerian-Lagrangian formulation:

$$
\begin{array}{rlrl}
\mathbf{u} & =-\nabla \phi \quad \text { in } \Omega_{F}(t) \\
\Delta \phi & =0 \quad \text { in } \Omega_{F}(t) \\
\frac{\partial \mathbf{R}}{\partial t} & =\mathbf{u} \quad & \text { on } \Gamma(t) \\
D_{t} \phi & =f \quad & \text { on } \Gamma(t),
\end{array}
$$

where $\mathbf{u}$ is the velocity field, $\phi$ the velocity potential and $D_{t}$ stands for the convective derivative, $D_{t} \phi=$ $\frac{\partial \phi}{\partial t}+\mathbf{u} \cdot \nabla \phi$. The function $f$ contains the contribution of the various forces acting on $\Gamma(t)$ and will be specified in 2.10. Let $p$ be the pressure field, $\rho$ the fluid density and $U$ the potential function of the body forces per unit mass (usually the gravitational forces) in $\Omega_{F}(t)$, excluding interface contact forces. Eqn. (2.4) is the dynamic boundary condition of $\phi$ on the free surface and comes from the conservation of momentum

$$
D_{t} \phi=-U+\frac{1}{2}|\mathbf{u}|^{2}-\frac{\left(p-p_{a}\right)}{\rho} \text { in } \Omega_{F}(t)
$$

imposing the continuity of the stress tensor on $\Gamma(t)$.

Depending upon the flow assumptions, some of the terms in the right hand side of (2.5) may be negligible compared to the others. For example, in the case of gravity wave propagation, see [10], the gravitational field $\mathbf{g}=-\nabla U$ has to be considered and surface tension forces on the free surface can be neglected. Alternatively, the Rayleigh-Taylor instability of an infinite jet is driven by surface tension and gravity terms can be neglected, see [1].

To include the effects of electric forces on the dynamics we assume that the fluid in $\Omega_{E}(t)$ is an insulator gas (or liquid) of permittivity $\epsilon$. In response to an electric field $\mathbf{E}$ applied to the system, the electric charges 
in $\Omega_{E}(t)$ do not flow but polarize, creating electric stresses acting on the liquid surface. The fluid in $\Omega_{F}(t)$ is considered a perfect conductor and charges flow instantaneously from the liquid bulk to the surface. The electric field is thus $\mathbf{E}=\mathbf{0}$ inside $\Omega_{F}(t)$ and $\Gamma(t)$ is an electric equipotential surface. Under these assumptions, $\mathbf{E}$ is solenoidal and can be expressed as the gradient of an electric potential field $V$ :

$$
\begin{array}{rlrl}
\mathbf{E} & =-\nabla V \quad \text { in } \Omega_{E}(t) \\
\Delta V & =0 \quad & \text { in } \Omega_{E}(t) \\
V & =0 & & \text { on } \Gamma(t),
\end{array}
$$

where, without loss of generality, the value of the electric potential on the moving surface has been set to zero. The electric stresses act only in the normal direction to $\Gamma(t)$ and compete against the restoring effects of surface tension. The pressure jump across the free surface $\Gamma(t)$ is given by

$$
p-p_{a}=\gamma \kappa-\frac{\epsilon}{2} E_{n}^{2}
$$

where $E_{n}=\mathbf{n} \cdot \nabla V$ and $\mathbf{n}$ the unit normal vector pointing from the interior to the exterior domain. The surface tension contribution is $\gamma \kappa$, where $\kappa$ is twice the mean curvature of the surface and $\gamma$ the surface tension coefficient. The presence of the electric field in the fluid dynamics is accounted for by simply adding the forces from the electrical stresses. Therefore, the function $f$ in Eqn. 2.4 is:

$$
f=\frac{1}{2}|\mathbf{u}|^{2}-\frac{1}{\rho}\left(\gamma \kappa-\frac{\epsilon}{2} E_{n}^{2}\right) .
$$

Note that gravity forces have been neglected as inertia, surface tension and electric forces are the dominant forces in the physical applications presented here.

To make the equations dimensionless we introduce the usual characteristic scales for length $r_{0}$, capillary time $t_{0}$ and electric potential $V_{0}$. The non-dimensional model equations remain except the equation for $f$, which becomes

$$
f=\frac{1}{2}|\mathbf{u}|^{2}-\kappa+\frac{B}{2} E_{n}^{2}
$$

where $B=\epsilon V_{0}^{2} /\left(\gamma r_{0}\right)$ is the non-dimensional electric Bond number which represents a normalized electrostatic pressure. In what follows all the variables are dimensionless. We have thus avoided a separate notation for the dimensionless variables.

We point out that a fluid with finite conductivity in $\Omega_{F}(t)$ could be assumed. This would require solving for the electric field $\mathbf{E}$ also in $\Omega_{F}(t)$ and accounting for the charge density jump across the free surface, feasible in the context of the Nitsche finite element method, 22, 23.

Next, we formulate the above Eulerian-Lagrangian equations in a complete Eulerian framework.

\subsection{The Eulerian formulation of the model equations}

Let $\Omega_{D}$ be a fixed three dimensional domain that will contain the free boundary for all times. In Fig. 2.1. $\Omega_{D}=\Omega_{F} \cup \Omega_{E}$. Eqns. (2.3) and (2.4), which are posed on a moving surface, can be reformulated in this fixed domain using the level set/extended potential technique described in [10, 11. In this approach, the moving front $\Gamma(t)$ and velocity potential $\left.\phi\right|_{\Gamma(t)}$ are embedded into functions $\Psi$ and $G$ of one higher dimension, respectively. The level set function $\Psi$ and the extended velocity potential function $G$ are defined on the fixed computational domain $\Omega_{D}$ that contains the free boundary for $t \in[0, T]$ and such that

$$
\begin{aligned}
& \Psi(\mathbf{R}(\mathbf{s}, t), t)=0 \\
& G(\mathbf{R}(\mathbf{s}, t), t)=\left.\phi\right|_{\Gamma(t)}
\end{aligned}
$$

for $t \in[0, T]$. Following the derivation in [11, Eqns. [2.3) and (2.4) transform into:

$$
\begin{aligned}
& \Psi_{t}+\mathbf{u}_{\mathrm{ext}} \cdot \nabla \Psi=0 \quad \text { in } \Omega_{D} \\
& G_{t}+\mathbf{u}_{\mathrm{ext}} \cdot \nabla G=f_{\mathrm{ext}} \quad \text { in } \Omega_{D},
\end{aligned}
$$


respectively. The subscript "ext" in Eqns. 2.14 and (2.15) denotes the extension of $f$ and $\mathbf{u}$ on the free boundary onto $\Omega_{D}$ (the details of how to perform these extensions will be explained Section 5.2). Note that here we have set $\Omega_{D}=\Omega_{F} \cup \Omega_{E}$, but it could be chosen in any other way. The only requirement is that the free boundary should always be included in $\Omega_{D}$.

The Eulerian model equations for the electrohydrodynamic problem are thus:

$$
\begin{aligned}
\mathbf{u} & =-\nabla \phi \quad \text { in } \Omega_{F}(t) \\
\Delta \phi & =0 \quad \text { in } \Omega_{F}(t) \\
\phi & =G \quad \text { on } \Gamma(t) . \\
\Psi_{t}+\mathbf{u}_{\text {ext }} \cdot \nabla \Psi & =0 \quad \text { in } \Omega_{D} \\
G_{t}+\mathbf{u}_{\text {ext }} \cdot \nabla G & =f_{\text {ext }} \quad \text { in } \Omega_{D} \\
\mathbf{E} & =-\nabla V \quad \text { in } \Omega_{E}(t) \\
\Delta V & =0 \quad \text { in } \Omega_{E}(t) \\
V & =0 \quad \text { on } \Gamma(t) .
\end{aligned}
$$

The boundary conditions for 2.19) and 2.20) can be set to $\mathbf{n}_{D} \cdot \nabla \Psi=0$ and $\mathbf{n}_{D} \cdot \nabla G=0$ respectively on $\partial \Omega_{D}, \mathbf{n}_{D}$ denoting the exterior normal to $\partial \Omega_{D}$. These assuptions mean that the level sets of $\Phi$ and $G$ will intersect $\partial \Omega_{D}$ orthogonally. The rest of the boundary conditions and domain geometries will be specified for each of the particular applications presented in this work.

The main advantage of the level set/extended potential formulation relies on the fact that any topological change of the free surface and evolving magnitudes within this boundary are directly taken into account by Eqns. 2.19 and 2.20). Moreover, the embedding provides a convenient regularization of possible singular geometries and prevents the blow up of diverging flow variables. This situation often occurs at fluid break-up.

\section{Numerical approximation methodology}

To explain the main ideas of the numerical method, it is sufficient to consider the fluid motion in $\Omega_{F}(t)$ given by Eqns. 2.16 to 2.20. The inclusion of the external electric field entails the solution of one more Laplace equation, namely Eqn. 2.22 and will be addressed in the electrohydrodynamic case study presented in Section 7. To construct the numerical approximation, we have to describe the time and space discretization of Eqns. 2.19), 2.20), the Nitsche solver for Eqn. 2.17), and the Nitsche-level set coupling. The study of the Nitsche finite element method in the context of time-dependent problems is one of the goals of this work, therefore it is presented in a separate section, Section 4 As some details of the level set method need the notation introduced in Section 4, a brief review of this method is postponed to Section 5

\subsection{Time discretization and the Nitsche-level set coupling}

The time discretization is done using a explicit forward Euler scheme with time step $\Delta t$. At each time step $t_{n}$, given $\Psi^{n}=\Psi\left(t_{n}\right)$ and $G^{n}=G\left(t_{n}\right)$ the following semi-discretized system has to be solved:

$$
\begin{aligned}
\mathbf{u}^{n} & =-\nabla \phi^{n} \quad \text { in } \Omega_{F}\left(t_{n}\right) \\
\Delta \phi^{n}(x, y, z) & =0 \quad \text { in } \Omega_{F}\left(t_{n}\right) \\
\left.\phi^{n}\right|_{\Gamma_{t_{n}}} & =\left.G^{n}\right|_{\Gamma_{t_{n}}} \\
\frac{\Psi^{n+1}-\Psi^{n}}{\Delta t} & =-\mathbf{u}_{\text {ext }}^{n} \cdot \nabla \Psi^{n} \quad \text { in } \Omega_{D} \\
\frac{G^{n+1}-G^{n}}{\Delta t} & =-\mathbf{u}_{\text {ext }}^{n} \cdot \nabla G^{n}+f_{\text {ext }}^{n} \quad \text { in } \Omega_{D} .
\end{aligned}
$$

Eqns. (3.1) to (3.3), which will be approximated using Nitsche's method, are strongly coupled to Eqns. (3.4) and (3.5), which are approximated by applying suitable finite difference level set schemes. First, the Dirichlet boundary condition $(3.3)$ is obtained from the spatial mesh values of $G^{n}$ in $\Omega_{D}$ and secondly $\mathbf{u}$ 
has to be provided from the finite element calculation to yield $\mathbf{u}_{\text {ext }}^{n}$ and $f_{\text {ext }}^{n}$ in the mesh points of $\Omega_{D}$. To facilitate the reading for those who would like to escape the technical details, the basics algorithm can be summarized in Alg. 3.1 .

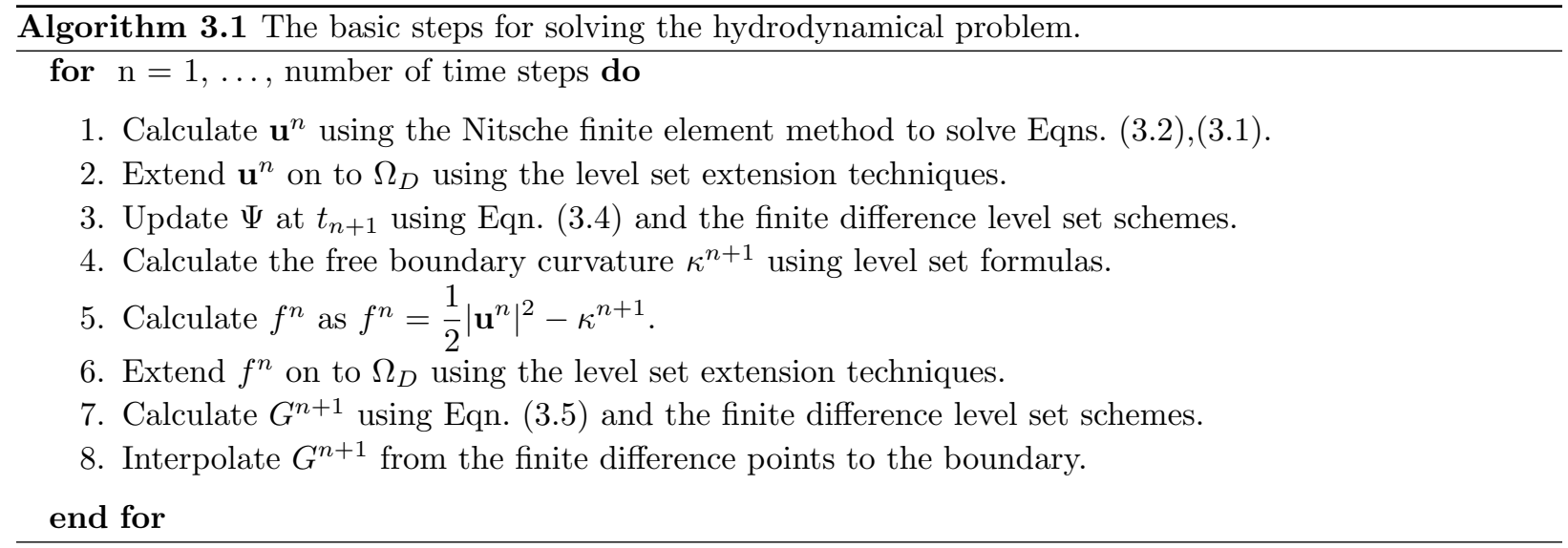

\subsection{Spatial discretization}

We will here briefly introduce the spatial discretization used by each numerical technique. A hexahedral finite element mesh is used for the finite element method while a finite difference mesh is used for the level set schemes. The finite element mesh will be described in detail in the next Section. A convenient feature of the present setup is that the vertices of the hexahedral mesh are used as points in the level set schemes. This is not necessary, but is used to simplify the data transfer between the two discretization methods. We set

$$
\Omega_{D}=\left[-L_{1}, L_{1}\right] \times\left[-L_{2}, L_{2}\right] \times\left[-L_{3}, L_{3}\right],
$$

where $L_{1}, L_{2}$ and $L_{3}$ are such that $\Omega_{D}$ will contain the free boundary for all $t \in[0, T]$. The boundary of $\Omega_{D}$ is denoted by $\partial \Omega_{D}$. Let be $\mathcal{P}_{D} \in \Omega_{D}$ the set of points used to define both meshes:

$$
\begin{aligned}
& \mathcal{P}_{D}=\left\{\left(x_{i}, y_{j}, z_{k}\right) \mid x_{i}=-L_{1}+i \Delta x, y_{j}=-L_{2}+j \Delta y, z_{k}=-L_{3}+k \Delta z,\right. \\
&\quad i=0, \ldots, N-1, j=0, \ldots, M-1, k=0, \ldots, L-1\},
\end{aligned}
$$

where $N, M$ and $L$ are the number of mesh points in the $x, y$ and $z$ directions, and $\Delta x=\frac{2 L_{1}}{N-1}, \Delta y=\frac{2 L_{2}}{M-1}$ and $\Delta z=\frac{2 L_{3}}{L-1}$ are the corresponding mesh sizes. Let $u, v, w$ be the free boundary velocity components in the $x, y, z$ directions respectively and $a=u_{\text {ext }}, b=v_{\text {ext }}, c=w_{\text {ext }}$ the corresponding extension onto $\Omega_{D}$. Let $G_{i, j, k}^{n}$ be the numerical approximation of the extended velocity potential $G\left(x_{i}, y_{j}, z_{k}, t_{n}\right)$. A first order upwind scheme approximation with a centered source term of Eqn. 3.5 yields, for $i=1, \ldots, N-2$, $j=1, \ldots, M-2$ and $k=1, \ldots, L-2$,

$$
\begin{aligned}
G_{i, j, k}^{n+1} & =G_{i, j, k}^{n}-\Delta t\left(\max \left(a_{i, j, k}^{n}, 0\right) D_{i, j, k}^{-x}+\min \left(a_{i, j, k}^{n}, 0\right) D_{i, j, k}^{+x}\right. \\
& +\max \left(b_{i, j, k}^{n}, 0\right) D_{i, j, k}^{-y}+\min \left(b_{i, j, k}^{n}, 0\right) D_{i, j, k}^{+y} \\
& \left.+\max \left(c_{i, j, k}^{n}, 0\right) D_{i, j, k}^{-z}+\min \left(c_{i, j, k}^{n}, 0\right) D_{i, j, k}^{+z}\right)+\Delta t f_{i, j, k}^{n},
\end{aligned}
$$

where $D_{i, j, k}^{-x}, D_{i, j, k}^{+x}$ are the backward and forward finite difference approximations for the derivative in the $x$ direction (same for the corresponding $y$ and $z$ backward and forward derivatives). The exact same scheme is used to approximate Eqn. (3.4).

The discrete boundary conditions on $\partial \Omega_{D}$, i.e. the six faces of the rectangular box $\Omega_{D}$, can be chosen in different ways depending upon the specific physics of the flow. For the applications presented here, we use a first order discretization of $\mathbf{n} \cdot \nabla G=0$ and $\mathbf{n} \cdot \nabla \Psi=0$.

Initial values of $G_{i, j, k}^{0}$ are obtained by extending $\left.\phi(x, y, z, 0)\right|_{\Gamma(0)}$. 

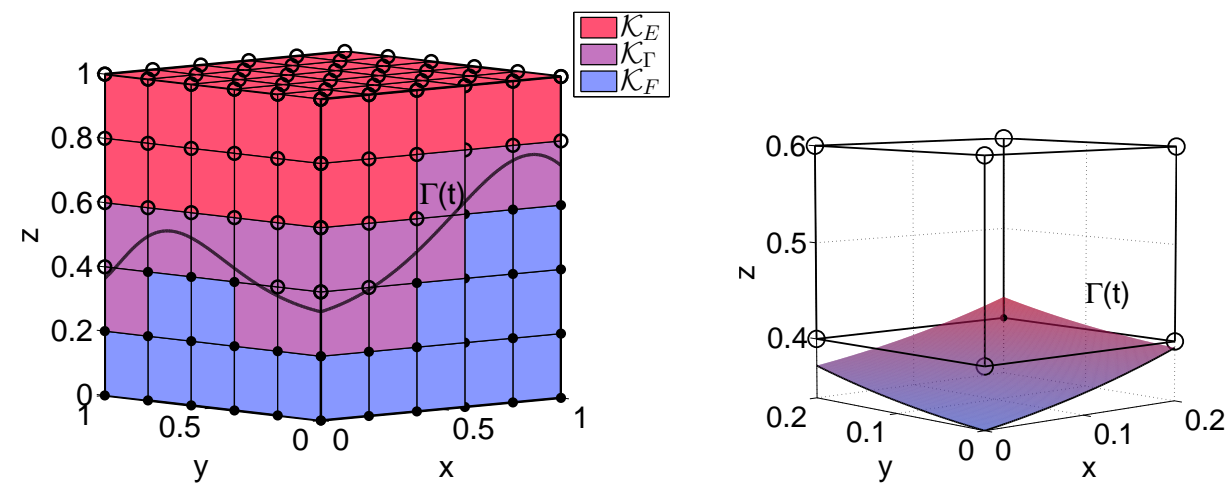

Figure 4.1: The mesh of the domain with $\Gamma(t)$ from Fig. 2.1 embedded in $\mathcal{K}_{D}$. The nodes are shared by the finite element and finite difference meshes. Filled circles are nodes below $\Gamma(t)$ and open circles are nodes above $\Gamma(t)$. Note that the mesh $\mathcal{K}_{\Gamma} \subset \mathcal{K}_{i}, i \in\{E, F\}$ contains elements with nodes both above and below $\Gamma(t)$. The element with nodes $(0,0,0.4)$ and $(0,0,0.6)$ in the left figure is in $\mathcal{K}_{\Gamma}$ since $\Gamma(t)$ is located as illustrated in the right figure.

\section{The Nitsche finite element method}

In a traditional finite element method, the computational domain is partitioned into a mesh which fits the boundary or interface, and the boundary conditions are enforced by appropriate function spaces. In contrast, Nitsche's method [15] is a consistent method for weakly enforcing the boundary conditions using the variational form. This means that the boundary conditions can be imposed without having the mesh fit the boundary, i.e. on a fixed background mesh. This is the motivation for using the Nitsche method here, since we then can use a single grid for both the level set methods and the finite element methods.

Here, the use of an unfitted method is challenging since we are interested in computing the free boundary velocity components $u, v, w$ as discussed in Section 3.2. This involves computing the full gradient of the solution to the Poisson problem Eqns. 2.17), 2.18) on the unfitted boundary. Therefore, computing the solution using a method that enforces the boundary condition by simple penalization will probably not work, since then the gradient on the boundary will likely be incorrect.

To use the Nitsche method, one must be able to compute certain integrals on the proper physical domain, as well as the interface. How this is done will be presented below. Furthermore, Nitsche's method may be written in symmetric and non-symmetric form (see 24), and we will here and in Section 6 illustrate why the non-symmetric form is crucial for computing the velocities on the boundary of the domain.

There is also a stability issue when the intersection of a finite element and the domain is small. To resolve this issue we use the ghost penalty stabilization [16. Below we investigate this stabilization numerically and see that it is preferred to the gradient jump stabilization given in [18].

\subsection{The method}

First we introduce some notation. Let $\mathcal{K}_{D}$ be fixed hexahedral partitioning of the computational domain $\Omega_{D}$ with vertices given by the set of points given in (3.7). Let the mesh size $h$ be the arithmetic mean of $\Delta x, \Delta y$ and $\Delta z$. The elements intersecting the domains $\Omega_{i}, i \in\{E, F\}$ form the meshes denoted by $\mathcal{K}_{i}$ and are given by

$$
\mathcal{K}_{i}=\left\{K \in \mathcal{K}_{D} \mid K \cap \Omega_{i} \neq \emptyset\right\} \quad i \in\{E, F\} .
$$

The elements which are cut by $\Gamma$ are of particular interest. These form a mesh denoted by $\mathcal{K}_{\Gamma}$ given by

$$
\mathcal{K}_{\Gamma}=\left\{K \in \mathcal{K}_{D} \mid K \cap \Gamma \neq \emptyset\right\} .
$$

Note that $\mathcal{K}_{\Gamma} \subset \mathcal{K}_{i}, i \in\{E, F\}$. See also Fig. 4.1 
Since the equations for the fluid potential $\phi$ and the electrical potential $V$ are the same (cf. 2.2 and (2.7), it is sufficient to present the finite element method for $\phi$. Furthermore, the spatial discretization is done on the semi-discretized Eqn. (3.2). For simplicity of notation, we will in this section drop the dependence on time and denote the boundary condition of $(3.3)$ by $g$. Therefore the general problem of interest can be written as

$$
\begin{array}{rlrl}
-\Delta \phi & =0 & & \text { in } \Omega_{F} \\
\phi=g & & \text { on } \Gamma .
\end{array}
$$

Now assume $\phi \in H^{1}\left(\Omega_{F}\right)$ and $g \in H^{1 / 2}(\Gamma)$, and let $\mathbb{Q}_{p}(K)$ be the space of tensor product polynomials of most order $p$ on an element $K$. Let the finite element space be

$$
\mathcal{V}_{h}=\left\{v_{h} \in H^{1}\left(\mathcal{K}_{F}\right)\left|v_{h}\right|_{K} \in \mathbb{Q}_{p}(K), \forall K \in \mathcal{K}_{F}\right\}
$$

with basis $\left\{N_{j}\right\}_{j=1}^{\mathcal{N}_{F}}$. Here we use trilinear elements, i.e. $p=1$. A general formulation of Nitsche's method with stabilization now reads: Find $\phi_{h} \in \mathcal{V}_{h}$ such that

$$
a_{h}\left(\phi_{h}, v\right)+s_{h}\left(\phi_{h}, v\right)=l_{h}(v) \quad \forall v \in \mathcal{V}_{h},
$$

where $a_{h}\left(\phi_{h}, v\right)$ and $l_{h}$ are the forms corresponding to the Nitsche method and $s_{h}\left(\phi_{h}, v\right)$ is a stabilization term which we will return to below.

To understand the main idea of Nitsche's method, we will first consider the symmetric form:

$$
\begin{aligned}
a_{h}\left(\phi_{h}, v\right) & =\int_{\Omega_{F}} \nabla \phi_{h} \cdot \nabla v d x-\underbrace{\int_{\Gamma} \mathbf{n} \cdot \nabla \phi_{h} v d s}_{\text {consistency }}-\underbrace{\int_{\Gamma} \phi_{h} \mathbf{n} \cdot \nabla v d s}_{\text {symmetry }}+\underbrace{\frac{\gamma_{D}}{h} \int_{\Gamma} \phi_{h} v d s}_{\text {coercivity }} \\
l_{h}(v) & =-\int_{\Gamma} g \mathbf{n} \cdot \nabla v d s+\frac{\gamma_{D}}{h} \int_{\Gamma} g v d s .
\end{aligned}
$$

We obtain the first two terms in (4.7) by the integration by parts of (4.3), where the boundary term is included for consistency. To obtain a symmetric form, we add the term labeled "symmetry" and a matching term in the right hand side, namely the first term of (4.8). The method is not coercive (even in the bodyfitted case) unless the coercivity term is added with a sufficiently large parameter $\gamma_{D}>0$. This term must also have a matching term in the right hand side, namely the second term of (4.8).

The idea of the non-symmetric method is similar. In this form, we do not add the corresponding symmetric term. Rather, we add the non-symmetric term corresponding to the consistency term. As shown in [24], coercivity is ensured also for $\gamma_{D}=0$, which means the coercivity term can be excluded. Thus, the non-symmetric method reads

$$
\begin{aligned}
a_{h}\left(\phi_{h}, v\right) & =\int_{\Omega_{F}} \nabla \phi_{h} \cdot \nabla v d x-\underbrace{\int_{\Gamma} \mathbf{n} \cdot \nabla \phi_{h} v d s}_{\text {consistency }}+\underbrace{\int_{\Gamma} \phi_{h} \mathbf{n} \cdot \nabla v d s}_{\text {non-symmetry }} \\
l_{h}(v) & =\int_{\Gamma} g \mathbf{n} \cdot \nabla v d s .
\end{aligned}
$$

In practice, the function $g$ is a trilinear function defined by the point values of $\left.G\right|_{\Gamma}$ in the finite difference grid. The normal $\mathbf{n}$ is given by the level set function (5.4).

To implement any of these methods one proceeds as usual: expand $\phi_{h}$ into the basis of $\mathcal{V}_{h}$ with coefficients $\Phi_{j}, j=1, \ldots, \mathcal{N}_{F}$, as

$$
\phi_{h}=\sum_{j=1}^{\mathcal{N}_{F}} \Phi_{j} N_{j} .
$$


The finite element forms 4.9 and 4.10 corresponds to a matrix $\mathbf{A}$ and vector $\mathbf{b}$, respectively, with entries

$$
\begin{aligned}
(\mathbf{A})_{i j} & =\int_{\Omega_{F}} \nabla N_{j} \cdot \nabla N_{i} d x-\int_{\Gamma}\left(\mathbf{n} \cdot \nabla N_{j} N_{i}-N_{j} \mathbf{n} \cdot \nabla N_{i}\right) d s \\
(\mathbf{b})_{i} & =\int_{\Gamma} g \mathbf{n} \cdot \nabla N_{i} d s .
\end{aligned}
$$

The corresponding matrix and vector for the terms in the symmetric form (4.7) and 4.8 are computed similarly.

\subsection{Stabilization techniques}

Nitsche's method breaks down if $K \cap \Omega_{F}$ is zero, since the bilinear form will not be coercive. Intuitively this can be understood by looking at the illustration on the right of Fig. 4.1. Here, the intersection $K \cap \Omega_{F}$ is zero, and thus the node with coordinates $(0,0,0.6)$ will be loosely coupled to the domain $\mathcal{K}_{F}$ since the integral over $\Omega_{F}$ will not contribute to this node. This also means that the diagonal element in the system matrix corresponding to this node will be zero and the corresponding system matrix will be singular. For this reason, we need a stabilization of the method by, for example, modifying the forms by adding $s_{h}\left(\phi_{h}, v\right)$ as in (4.6). We have investigated two different stabilization terms: the ghost penalty stabilization [16] and a gradient jump stabilization [18. The former will be presented in detail below, because this is found to be the preferred method.

Since we use hexahedral elements, the implementation of the two stabilization types is straightforward. For meshes with simplicial elements, only the gradient jump stabilization is straightforward. Worth mentioning is that in the setting of discontinuous finite elements, no modification of the variational form is needed, since it is possible to do a simple element-wise association, regardless of element geometry, see [20]. Since discontinuous Galerkin methods are often preferred to continuous Galerkin for hyperbolic problems, this would mean that it is indeed possible and perhaps advantageous to use the same discretization technique for both the hyperbolic level set equations and the elliptic Laplace problems.

\subsubsection{Ghost penalty stabilization}

Following [16], the ghost penalty stabilization is as follows. Let $\omega_{K} \subset \mathcal{K}_{F}$ be a patch around an element $K \in \mathcal{K}_{\Gamma}$ with sufficient size to maintain stability but not too large to compromise accuracy. A precise definition can be found in [16. Furthermore, let $\pi_{\omega_{K}}$ be the $L^{2}$ projection given by

$$
\pi_{\omega_{K}}: L^{2}\left(\omega_{K}\right) \rightarrow \mathbb{Q}_{p}\left(\omega_{K}\right)
$$

The stabilization term reads

$$
s_{h}\left(\phi_{h}, v\right)=\sum_{K \in \mathcal{K}_{\Gamma}} \int_{\omega_{K}} \frac{\gamma_{g p}}{h^{2}}\left(\phi_{h}-\pi_{\omega_{K}} \phi_{h}\right) v d x \quad \forall v \in \mathcal{V}_{h},
$$

where $\gamma_{g p}>0$ is a stabilization term. Note that the term is inconsistent since $\mathbb{Q}_{p}\left(\omega_{K}\right) \notin \mathcal{V}_{h}$. The factor $h^{-2}$ is to make $s_{h}\left(\phi_{h}, v\right)$ scale with the same order as the other terms in 4.6) during mesh refinement.

In practice we form $\omega_{K}$ by looking in the $3 \times 3 \times 3$ neighborhood of an element $K \in \mathcal{K}_{\Gamma}$. It is likely that this neighborhood will contain less than 27 elements, since we look for neighbors to an element on the boundary. We use three different element configurations to construct $\omega_{K}$. First, we try to form a patch composed of a $2 \times 2 \times 2$ block mesh as in Fig. 4.2. There are up to 8 possibilities of doing this and we choose the configuration with largest intersection with $\Omega_{F}$. However, depending on how $\Gamma$ cuts $\mathcal{K}_{F}$, forming a $2 \times 2 \times 2$ patch is not always possible. If this is the case, we form patches of size $2 \times 2 \times 1$, or sometimes even $2 \times 1 \times 1$ elements. This latter configuration can for example occur when $K$ is touching $\partial \Omega_{D}$. Note that $\omega_{K}$ contains the full hexahedral elements. We are not considering the geometry of the cut element $K \cap \Omega_{F}$ when considering $\omega_{K}$. 
We now turn to the evaluation of $s_{h}\left(\phi_{h}, v\right)$. First note that $\pi_{\omega_{K}} \phi_{h}$ is defined by

$$
\int_{\omega_{K}} \pi_{\omega_{K}} \phi_{h} v d x=\int_{\omega_{K}} \phi_{h} v d x \quad \forall v \in \mathbb{Q}_{p}\left(\omega_{K}\right) .
$$

Let $\left\{M_{j}\right\}_{j=1}^{\mathcal{N}_{\omega_{K}}}$ be a basis for $\mathbb{Q}_{p}\left(\omega_{K}\right)$ such that

$$
\pi_{\omega_{K}} \phi_{h}=\sum_{j=1}^{\mathcal{N}_{\omega_{K}}} \Pi_{j} M_{j} .
$$

The unknown coefficients $\Pi_{j}, j=1, \ldots, \mathcal{N}_{\omega_{K}}$ can be written in terms of the $\mathcal{N}_{F}$-vector $\Phi$ (cf.4.11) by noting that the projection 4.16) is equivalent to the $\mathcal{N}_{\omega_{K}} \times \mathcal{N}_{\omega_{K}}$ linear system

$$
\mathbf{M}_{\omega} \Pi=\mathbf{d},
$$

where

$$
\left(\mathbf{M}_{\omega}\right)_{i j}=\int_{\omega_{K}} M_{j} M_{i} d x
$$

and using 4.11

$$
\begin{aligned}
(\mathbf{d})_{i} & =\int_{\omega_{K}} \phi_{h} M_{i} d x \\
& =\int_{\omega_{K}} \sum_{k=1}^{\mathcal{N}_{F}} \Phi_{k} N_{k} M_{i} d x \\
& =(\mathbf{R} \Phi)_{i}
\end{aligned}
$$

with

$$
(\mathbf{R})_{i k}=\int_{\omega_{K}} N_{k} M_{i} d x .
$$

Now we are able to state 4.15 in terms of matrix-matrix and matrix-vector multiplications. For the first part of 4.15 we have, with $v=N_{i}$,

$$
\int_{\omega_{K}} \phi_{h} N_{i} d x=\int_{\omega_{K}} \sum_{j=1}^{\mathcal{N}_{F}} \Phi_{j} N_{j} N_{i} d x
$$

and we see that we need the mass matrix $\mathbf{M}$ given by

$$
(\mathbf{M})_{i j}=\int_{\omega_{K}} N_{j} N_{i} d x .
$$

The size of this matrix depends on the number of nodes in the patch.

For the second term of (4.15), we have using (4.17)

$$
\int_{\omega_{K}} \pi_{\omega_{K}} \phi_{h} N_{i}=\int_{\omega_{K}} \sum_{k=1}^{\mathcal{N}_{\omega_{K}}} \Pi_{k} M_{k} N_{i} d x
$$

which using Eqn. 4.23 corresponds to

$$
\begin{aligned}
\mathbf{R}^{T} \Pi & =\mathbf{R}^{T} \mathbf{M}_{\omega}^{-1} \mathbf{d} \\
& =\mathbf{R}^{T} \mathbf{M}_{\omega}^{-1} \mathbf{R} \Phi .
\end{aligned}
$$




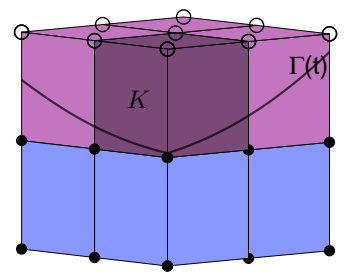

Figure 4.2: A patch $\omega_{K}$ formed by the $2 \times 2 \times 2$ neighborhood of element $K$.

The matrix $\mathbf{S}$ corresponding to the stabilization term 4.15 thus reads

$$
\mathbf{S}=\sum_{K \in \mathcal{K}_{\Gamma}} \frac{\gamma_{g p}}{h^{2}}\left(\mathbf{M}-\mathbf{R}^{T} \mathbf{M}_{\omega}^{-1} \mathbf{R}\right) .
$$

Since we use trilinear basis functions, $\mathcal{N}_{\omega_{K}}=8$ and thus $\mathbf{M}_{\omega}$ is an $8 \times 8$ positive definite matrix which is to be inverted for each patch. Furthermore, if $\omega_{K}$ is of size $2 \times 2 \times 2$, then $\mathbf{R}$ is effectively a $8 \times 27$-matrix and $\Phi$ effectively a 27 -vector since all but 27 basis functions of $\mathcal{V}_{h}$ are non-zero. We conclude by noting that this stabilization term is in general difficult to construct on simplicial meshes.

\subsubsection{Gradient jump stabilization}

Another stabilization possibility includes adding the jumps of the gradients on the faces of the elements in $\mathcal{K}_{\Gamma}$ [18. Let this set of faces be denoted by $\mathcal{F}_{\Gamma}$, and let the boundary faces of $\mathcal{K}_{F}$ be excluded from $\mathcal{F}_{\Gamma}$ (see 18 for a precise definition).

The gradient jump stabilization reads

$$
s_{h}\left(\phi_{h}, v\right)=\sum_{F \in \mathcal{F}_{\Gamma}} \int_{F} \gamma_{g j} h^{\alpha}\left[\mathbf{n}_{F} \cdot \nabla \phi_{h}\right]\left[\mathbf{n}_{F} \cdot \nabla v\right] d s
$$

where $\gamma_{g j}>0$ is a stabilization parameter and $[w]=w^{+}-w^{-}$, with $w^{ \pm}(\mathbf{x})=\lim _{t \rightarrow 0^{+}} w\left(\mathbf{x} \mp t \mathbf{n}_{F}\right)$ denoting the jump of $w$ across the face $F$ with normal $\mathbf{n}_{F}$. Furthermore, $\alpha=3$ for the non-symmetric method and $\alpha=1$ for the symmetric method. Note that the jump is computed over the full face $F$, even though the element may be cut by $\Gamma$.

This is a common stabilization for simplicial meshes and is related to the classical continuous interior penalty method. The implementation is straightforward given a quadrature rule on the faces. Here a quadrature rule for a $2 \mathrm{D}$ quadrilateral is used, which is mapped to the $3 \mathrm{D}$ faces.

\subsection{Approximation of integrals}

The integrals in the Nitsche parts $a_{h}\left(\phi_{h}, v\right)$ and $l_{h}(v)$ of $(4.6)$ are to be computed over $\Omega_{F}$ and $\Gamma$, which are not aligned with the finite element mesh $\mathcal{K}_{F}$. This is in contrast to the integrals in the stabilization term $s_{h}\left(\phi_{h}, v\right)$, where the integrals are computed over the full hexahedral volumes or faces. To perform the volume integrals on the cut elements of $\mathcal{K}_{\Gamma}$, we create a sub-partitioning of tetrahedra using the marching cubes algorithm [25] assuming a trilinear approximation of $\Gamma$. Similarly, we create a triangulation to perform the integrals over $\Gamma$. This can be a costly procedure, but we make use of the following:

- The partitions are local on each element, which means that the construction can be trivially parallelized. No connectivity is needed.

- The partitions are only used for setting up the quadrature rules. Since the finite element basis functions are not mapped, the quality of these partitions is not important for accuracy. 
Other methods for obtaining quadrature points are possible. For example the marching tetrahedron algorithm [26], which yields more simplices and thus possibly improved accuracy. In some cases it is possible to write the integrand as a divergence-free function and use the divergence theorem to perform the integrals on manifolds of one (or more) less dimension, see for example [27].

We investigated increasing the accuracy by constructing the simplices on an octree-splitting of each element, but this had little effect on the global dynamics. It may also be possible to have other polyhedra than tetrahedra and triangles.

To illustrate the integration algorithm, we note that the integrals of 4.6) are of the form $\int_{\Omega_{F}} m(\mathbf{x}) d x$ or $\int_{\Gamma} m(\mathbf{x}) d s$ for some function $m(\mathbf{x})$. For simplicity we can thus refer to these terms as being of the form $\int_{Y} m(\mathbf{x}) d y$ with appropriate $m(\mathbf{x}), Y$ and $d y$. Following standard finite element techniques, each integral is computed element-wise over each $K \in \mathcal{K}_{F}$ but we must take special care for $K \in \mathcal{K}_{\Gamma}$. On each element $K \in \mathcal{K}_{\Gamma}$ we partition $K \cap Y$ into a set of local simplices $\tau_{K}$ (tetrahedra for the volume integrals or triangles for the surface integrals) such that

$$
\int_{K \cap Y} m(\mathbf{x}) d y \approx \sum_{T \in \tau_{K}} \int_{T} m(\mathbf{x}) d y .
$$

This cannot be made exact unless the computational domain is exactly represented by $\tau_{K}$.

The integral 4.31 is computed using standard techniques: Each simplex $T$ is mapped to the reference simplex $\hat{T}$ on which we have $\mathcal{N}_{q}$ Gaussian quadrature points and weights denoted by $\left\{\xi_{j}\right\}_{1}^{\mathcal{N}_{q}}$ and $\left\{w_{j}\right\}_{1}^{\mathcal{N}_{q}}$ respectively. Each integral is now computed by the formula

$$
\int_{T} m(\mathbf{x}) d y \approx \sum_{j=1}^{\mathcal{N}_{q}} m\left(\mathbf{x}\left(\xi_{j}\right)\right)\left|J\left(\xi_{j}\right)\right| w_{j},
$$

where $\xi \rightarrow \mathbf{x}(\xi)$ is the map of $\hat{T}$ to $T$ and $|J|$ the determinant of the Jacobian of this map. Since we use linear simplices, this is constant. Note that any gradient operators in $m(\mathbf{x})$ are not mapped, as would be the case if the whole finite element would be mapped.

\subsection{Numerical tests of the Nitsche methods}

Two types of Nitsche methods are presented above: the non-symmetric (cf. (4.9), (4.10) ) and the symmetric (cf. (4.7), (4.8)) forms. Two stabilization types are also presented: the ghost penalty stabilization (4.15) and the gradient jump stabilization 4.30). The literature provides theory and numerical results for the condition number behavior as well as the errors of the Poisson problem in $L^{2}$ and energy norms (cf. [16, 18, 28, and the references therein). However, we are interested in the maximum error of the full gradient of $\phi_{h}$ on the boundary $\Gamma$, i.e., the error in the norm

$$
\|v\|=\max _{\substack{\mathbf{x} \in \Gamma \\ j=1,2,3}}\left|\frac{\partial v}{\partial x_{j}}(\mathbf{x})\right| .
$$

We now report numerically investigations of the performance of the Nitsche methods with respect to this norm. The theoretical investigation for this error is beyond the scope of this paper. Rather, three studies will be performed for both the non-symmetric and the symmetric methods:

- Dependence of $\|\left|\phi-\phi_{h}\right|||$ on the parameters $\gamma_{g p}, \gamma_{g j}$ and $\gamma_{D}$.

- Convergence properties with respect to $\left\|\phi-\phi_{h}\right\|$.

- Dependence of $\left\|\phi-\phi_{h}\right\| \mid$ on the position of $\Omega_{F}$ in the background mesh $\mathcal{K}_{D}$.

The main motivation for this study is to give some motivation as to which method to use in the electrohydrodynamical computations (cf. Section 6, where will investigate the Nitsche methods in the hydrodynamical 


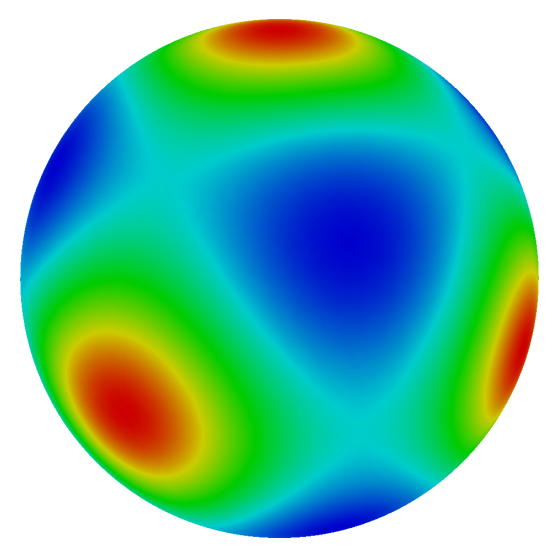

Figure 4.3: The exact solution $\phi$.

context). We here consider the problem (4.3), 4.4 with $\Omega_{F}$ being the sphere $\Omega_{F}=\left\{\mathbf{x} \in \mathbb{R}^{3}:\|\mathbf{x}-\mathbf{c}\| \leq 1\right\}$. We let the center position be $\mathbf{c}=\mathbf{0}$ (except in Section 4.4 .3 . The sphere is placed in the box $\Omega_{D}=[-1,1]^{3}$ and the exact solution is given by

$$
\phi(x, y, z)=e c(x, y)+e c(y, x)+e c(x, z)+e c(z, x)+e c(y, z)+e c(z, y)
$$

where $e c$ is the harmonic function

$$
e c(\xi, \eta)=e^{\xi^{2}-\eta^{2}} \cos (2 \xi \eta)
$$

Fig. 4.3 illustrates $\phi$. The exact gradients are straightforwardly computed, giving $\|\phi\|=9.1535$.

All linear systems are solved using GMRES with an incomplete LU factorization as preconditioner in the PETSc [29] framework with a relative convergence tolerance (i.e. a measure of the relative decrease in the preconditioned residual norm) of $10^{-8}$.

\subsubsection{Parameter dependence}

One practical advantage of the non-symmetric method is that $\gamma_{D}=0$. Thus, the parameter study for this method will only consider the stabilization parameters $\gamma_{g p} 4.15$ and $\gamma_{g j} 4.30$. Fig. 4.4 shows the error $\left\|\phi-\phi_{h}\right\| \mid$ as a function of parameter values for meshes with $h=0.2,0.1,0.05,0.025$ and 0.0125 (top to bottom). We observe that the range of the $y$-axis is rather small, particularly for the finer meshes (for the relative error, recall that $\|\phi \mid\|=9.1535)$. Thus we may conclude that for this particular example, computing $\| \phi-\phi_{h}||$ using the non-symmetric Nitsche method is not particularly sensitive to either $\gamma_{g p}$ or $\gamma_{g j}$.

For the parameter study of the symmetric method we study $\gamma_{D}=\{1,2,5,10,20,30, \ldots, 100\}$ and $\gamma_{i} \in$ $\{0.01,0.02,0.03, \ldots, 0.1,0.2,0.3, \ldots, 2\}, i=\{g p, g j\}$ using a fixed mesh with $N=81$ corresponding to $h=0.025$. The results are illustrated in Fig. 4.5. The ghost penalty stabilization gives us the smallest as well as the largest errors. Small $\gamma_{D}$ values causes the algebraic system to fail to converge and is thus not illustrated, and some small parameter values clearly give very large errors. We have algebraic convergence for the ghost penalty stabilization only when $\gamma_{D}$ is sufficiently large, although a large $\gamma_{g p}$ value can assist. Nevertheless we see that a large $\gamma_{D}$ and a small stabilization parameter value are desirable for achieving low $\left\|\phi-\phi_{h}\right\| \mid$ values.

\subsubsection{Convergence results}

When investigating the convergence of $\left\|\phi-\phi_{h}\right\| \mid$ with respect to $h$, we take advantage of the study in Section 4.4.1 for choosing suitable parameters. For the non-symmetric method, we select $\gamma_{g p} \in\{0.01,0.1,1,10\}$ and $\gamma_{g j}=\{0.1,1,10,100\}$. We obtain almost identical errors for the different stabilization methods except for the largest stabilization parameters, which gives slightly better convergence rate (1.3 vs 1.1 for both 


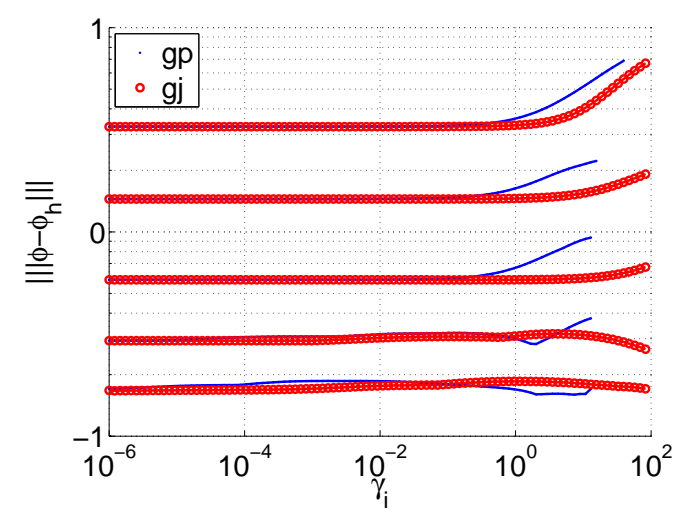

Figure 4.4: The error $\left\|\phi-\phi_{h}\right\| \mid$ of the non-symmetric method as a function of the stabilization parameter $\gamma_{g p}$ for ghost penalty stabilization ("gp") and as a function of $\gamma_{g j}$ for the gradient jump stabilization ("gj"). The mesh sizes are from top to bottom $h=0.2,0.1,0.05,0.025$ and 0.0125 .
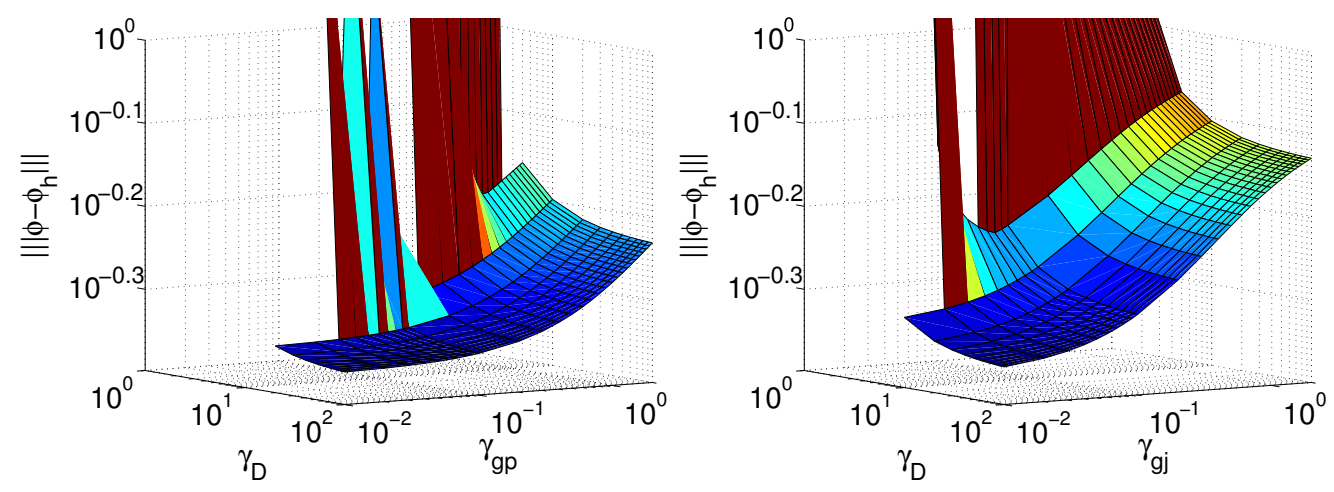

Figure 4.5: The error $\left\|\phi-\phi_{h}\right\| \mid$ given by the symmetric method as a function of $\gamma_{D}$ and $\gamma_{g p}$ for the ghost penalty stabilization (left) and of $\gamma_{D}$ and $\gamma_{g j}$ for the gradient jump stabilization (right). 

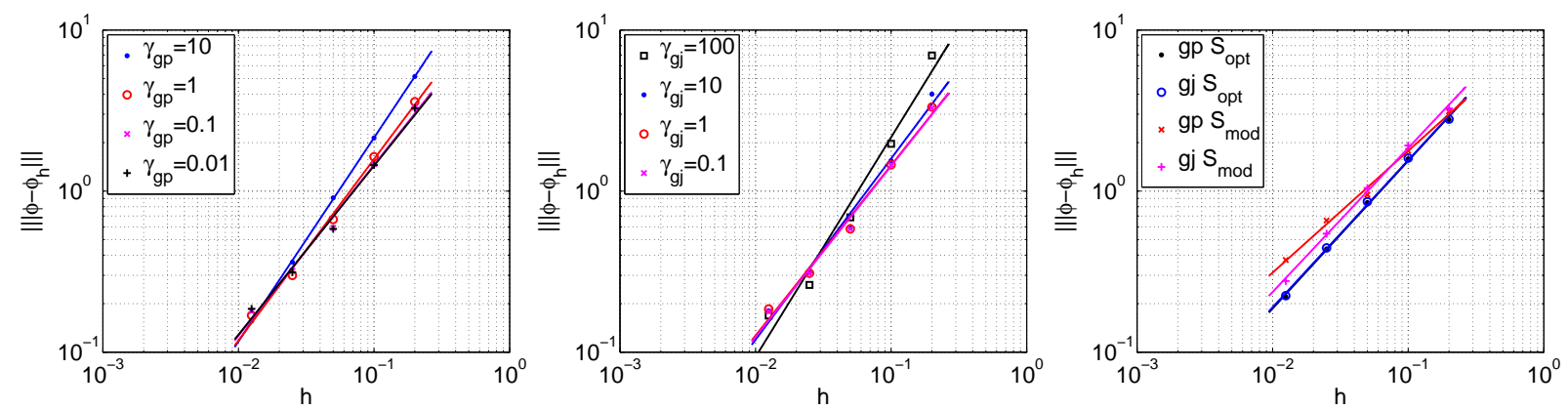

Figure 4.6: The value of $\left\|\mid \phi-\phi_{h}\right\| \|$ using the non-symmetric (left and middle) and symmetric (right) methods with ghost penalty stabilization ("gp") and gradient jump stabilization ("gj") for various parameter values.
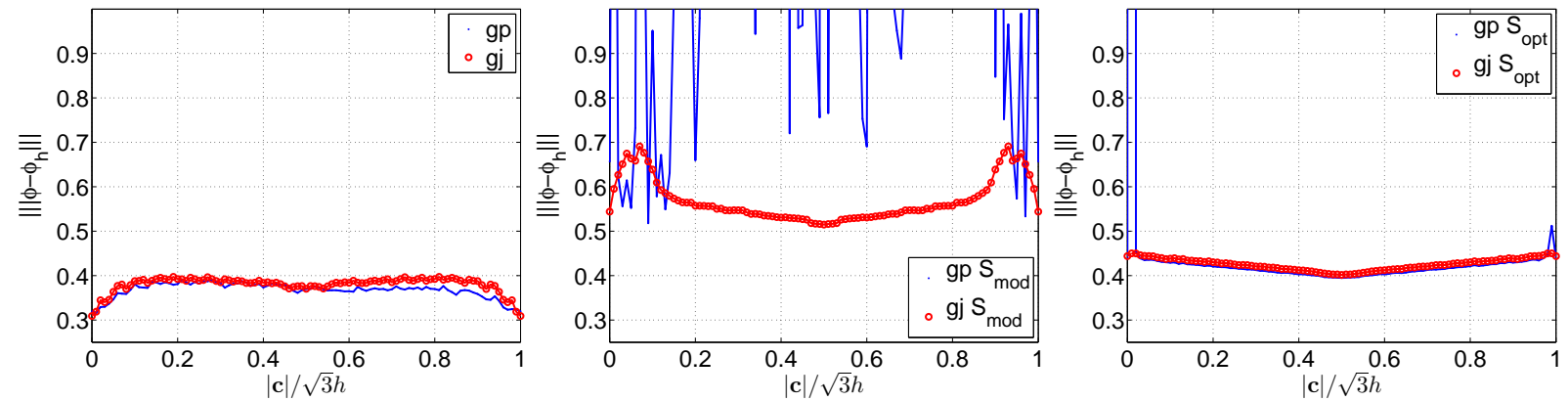

Figure 4.7: The value of $\left\|\left|\phi-\phi_{h} \|\right|\right.$ as a function of the center position for the non-symmetric method (left) and the symmetric method (middle and right). "gp" means ghost penalty stabilization and "gj" means gradient jump stabilization.

ghost penalty and gradient jump stabilization terms). Nevertheless, choosing different stabilization parameter values does not improve or worsen the convergence properties significantly. This is in accordance to the parameter study above.

For studying the symmetric method we choose two sets of parameters, namely the "optimal" set $S_{\text {opt }}=$ $\left\{\gamma_{g p}, \gamma_{g j}, \gamma_{D}\right\}=\{0.01,0.01,100\}$ as suggested by Fig. 4.5, and the "moderate" set $S_{\bmod }=\left\{\gamma_{g p}, \gamma_{g j}, \gamma_{D}\right\}=$ $\{0.1,0.1,10\}$. The results for the symmetric method are illustrated in the rightmost figure of Fig. 4.6. Here we see almost identical results for $S_{\text {opt }}$ with slope 0.91 . For $S_{\text {mod }}$ the gradient jump stabilization performs better (slope 0.89 ) than the ghost penalty stabilization (slope 0.76 ).

\subsubsection{Center position dependence}

In the above examples, the sphere center is set to $\mathbf{c}=\mathbf{0}$, which also the position of the lower left corner of a certain hexahedron. We now let c vary diagonally to the top right corner of this hexahedron and see how the error changes. We use a mesh with $N=81$. The left graph of Fig. 4.7 shows the error as a function of $|\mathbf{c}|$ normalized to the element size $h=0.025$ for the non-symmetric method with the two stabilization terms using $\gamma_{g p}=1$ and $\gamma_{g j}=10$. The middle and right graph of Fig. 4.7 show the corresponding results using the symmetric method with parameter sets $S_{\text {mod }}$ and $S_{\text {opt }}$ respectively.

The non-symmetric method can be seen to give errors that are independent of the position of the sphere in the background mesh, and both stabilization terms give quite similar results. This is similar to what was observed in Sections 4.4.1 and 4.4 .2 above. On the contrary, the symmetric method with gradient jump stabilization performs better than with ghost penalty stabilization. This is true for both $S_{\text {mod }}$ and $S_{\text {opt }}$. We can also see that small stabilization values cannot always control the errors in $\left\|\phi-\phi_{h}\right\|$.

The reason for the asymmetry when using ghost penalty stabilization is because the patches $\omega_{K}$ are not necessarily symmetrically placed. 


\subsubsection{Conclusions}

The errors $\left\|\phi-\phi_{h}\right\| \mid$ computed using the symmetric Nitsche method seem sensitive to the stabilization parameters $\gamma_{g p}$ and $\gamma_{g j}$, cf. Fig. 4.5. In addition, one must also select the parameter for coercivity $\gamma_{D}$. Thus it may be more difficult to find a suitable parameter set that will work in general when using this method. The symmetric method proved unsatisfactory due to failure when examining the center position dependence (cf. the right graph of Fig. 4.7). This is also a result of difficulty chosing suitable method parameters.

In contrast, the non-symmetric Nitsche method does not seem sensitive to variations in stabilization parameters $\gamma_{g p}$ and $\gamma_{g j}$, cf. Figs. 4.4 and 4.7 (left). The two different stabilization terms yield similar results in the convergence tests as shown in the left and middle graphs of Fig. 4.6 . Furthermore, the non-symmetric method seems to result in smaller errors than the symmetric method (cf. Figs. 4.6 and 4.7). The reason for this is likely the lack of the Nitsche parameter: The symmetric method is indeed optimal and consistent but the penalization-type enforcement of the boundary condition in the symmetric method influences the accuracy of the errors $\left\|\phi-\phi_{h}\right\|$.

Regarding the stabilization terms we intuitively expect the ghost penalty stabilization to be more stable compared to the gradient jump stabilization, since the stabilization region in the former is larger than in the latter (it takes data from the whole patch $\omega_{K}$ into account). The gradient jump stabilization term only obtains immediate information from the elements sharing a face. As mentioned above, the non-symmetric method does not seem to be sensitive to the choice of stabilization term. This is in contrast to the symmetric method, where the gradient jump stabilization seems preferred.

\section{The level set method}

The level set method is a widely used mathematical tool, developed by Osher and Sethian [30 to follow interfaces which move with a given velocity field $\mathbf{u}$. Let be $\Gamma(t)$ the set of points lying in the surface boundary at time $t$. This surface is defined by the zero level set of the scalar field $\Psi(\mathbf{x}, t)$ :

$$
\Gamma(t)=\{\mathbf{x} \mid \Psi(\mathbf{x}, t)=0\} .
$$

If we take a fixed $3 \mathrm{D}$ domain $\Omega_{D}$ that contains the free surface for all times, the initial value problem for the level set function $\Psi$ posed on $\Omega_{D}$ is

$$
\begin{aligned}
\Psi_{t}+\mathbf{u} \cdot \nabla \Psi & =0 \quad \text { in } \Omega_{D} \\
\Psi(\mathbf{x}, 0) & =s(\mathbf{x}) d(\mathbf{x}) \text { in } \Omega_{D},
\end{aligned}
$$

where $d(\mathbf{x})$ is the distance from the point $\mathbf{x}$ to the surface at the initial configuration $\Gamma(0), s(\mathbf{x})=-1$ if $\mathbf{x} \in \Omega_{F}(0)$ and $s(\mathbf{x})=+1$ if $\mathbf{x} \notin \Omega_{F}(0)$. Note that Eqn. (5.2) moves all the level sets of $\Psi$, not just the zero level set. Through the construction of extension velocities (see [31]), the velocity field on the interface can be suitably extended off of the interface so that the signed distance representation of the level set function is essentially maintained as the flow evolves. In the flows under study here, the velocity field $\mathbf{u}$ is calculated throughout $\Omega_{F}(t)$ as well as on $\Gamma(t)$, but only $\left.\mathbf{u}\right|_{\Gamma(t)}$ will be used to obtain the extension velocity field $\mathbf{u}_{\text {ext }}^{n}$. It follows that we need efficient and accurate methods to extend magnitudes off the front and to initialize (or re-initialize) the level set function: these will be discussed below. The free surface representation (5.1) allows the computation of geometric related variables through the level set function, as the normal vector to $\Gamma(t)$ and the curvature:

$$
\begin{aligned}
& \mathbf{n}=\frac{\nabla \Psi}{|\nabla \Psi|} \\
& \kappa=\nabla \cdot \mathbf{n} .
\end{aligned}
$$

The computation of the free surface curvature needed to calculate $f_{\text {ext }}$ in Eqn. 2.20 is done using (5.5). In practice we use a tricubic approximation [32] of $\Psi$ on $\mathcal{K}_{\Gamma}$ when we need to evaluate $\mathbf{n}$ and $\kappa$ in $\mathcal{K}_{\Gamma}$. Next we explain the initialization (re-initialization) and extension details. 


\subsection{Initialization}

For the level set framework to be accurate, it is important that the level set function $\Psi(\mathbf{x}, t)$ is a signed distance function throughout the computation. Initial construction of $\Psi(\mathbf{x}, 0)$ is usually not problematic since it is a computation that can be performed beforehand. However, under the evolution of (2.14), the signed distance property may fail. Thus a process of reinitialization is needed, which is a procedure of updating $\Psi(\mathbf{x}, t)$ such that it is a signed distance function. The difficulty with reinitialization is to maintain the zero level set, and thus avoid of volume conservation failure.

Here we use a method proposed by Chopp 32 which interpolates the nodal values of $\Psi(\mathbf{x}, t)$ to a tricubic function close to $\Gamma(t)$, giving good preservation of $\Gamma(t)$ during reinitialization and providing the foundation for a algorithm that is second order accurate. A brief explanation of the algorithm is as follows. Given a point $\mathbf{x}$, we seek $\mathbf{x}_{\Gamma} \in \Gamma(t)$ which is the closest point to $\mathbf{x}$ such that the following two conditions are fulfilled:

$$
\begin{aligned}
\Psi\left(\mathbf{x}_{\Gamma}, t\right) & =0 \\
\nabla \Psi\left(\mathbf{x}_{\Gamma}, t\right) \times\left(\mathbf{x}-\mathbf{x}_{\Gamma}\right) & =0 .
\end{aligned}
$$

The first condition requires $\mathbf{x}_{\Gamma}$ on the front and the second condition requires $\mathbf{x}-\mathbf{x}_{\Gamma}$ to be parallel to $\mathbf{n}$ at $\mathbf{x}_{\Gamma}$ (cf. (5.4)). This nonlinear system can be solved using a two-step Newton method, see 32 for details, and the level set function can be reconstructed by setting $\Psi(\mathbf{x}, t)=\operatorname{sgn}(\Psi(\mathbf{x}, t))\left|\mathbf{x}-\mathbf{x}_{\Gamma}\right|$. In practice we perform this computation on the nodes of the elements in $\mathcal{K}_{\Gamma}$. As will be discussed below, the closest points are crucial for constructing extended quantities accurately.

Away from the front we run a heap-based fast marching method in a narrow band (see [33) to solve the Eikonal equation $|\nabla \Psi(\mathbf{x}, t)|=1$. This is performed in conjunction with the construction of the extended quantities, which is discussed below. Typically we reinitialize $\Psi(\mathrm{x}, t)$ every 50 th time step and have a narrow band of width 10 elements.

\subsection{Extension}

In many physical applications the velocity and other variables of interest are only defined on the moving surface. To evolve equations in the non-physical domain $\Omega_{D}$ we need to extend magnitudes off the front. Let $m=m(\mathbf{x})$ be a magnitude defined on a smooth front. The only requirement to construct $m_{\text {ext }}=m_{\text {ext }}(\mathbf{x})$ is that

$$
\left.m_{\text {ext }}\right|_{\Gamma(t)}=m
$$

Furthermore, we note that to accurately compute geometric properties of the free boundary (such as the normal and curvature), it is important to maintain the level set function $\Psi(\mathbf{x}, t)$ as a signed distance function. One way to achieve this requirement in combination with 5.8 is to build $m_{\text {ext }}$ such that $\nabla m_{\text {ext }} \cdot \nabla \Psi=0$. Here this is done using the fast marching method, see [31.

It is important for the accuracy of this rebuilding that the initial data be accurate. Recall that from the initialization procedure described above, we have for any point $\mathbf{x} \in \mathcal{K}_{\Gamma}$ the closest point $\mathbf{x}_{\Gamma} \in \Gamma(t)$ using a tricubic interpolation of $\Psi(\mathbf{x})$ as discussed above. In practice, we find the closest points to the nodes of $\mathcal{K}_{\Gamma}$ which yields accurate initial data for the fast marching of $m$ by simply replacing the nodal value with the value of $m\left(\mathbf{x}_{\Gamma}\right)$.

How $m\left(\mathbf{x}_{\Gamma}\right)$ is evaluated depends on the representation of $m$ :

- If $m$ is a finite element function such as $\mathbf{u}=\nabla \phi_{h}$, we expand $\phi_{h}$ in its basis (as done in Eqn. (4.11)), compute the gradients of the basis functions and evaluate the sum $\nabla \phi_{h}\left(\mathbf{x}_{\Gamma}\right)=\sum_{j=1}^{\mathcal{N}_{F}} \Phi_{j} \nabla N_{j}\left(\mathbf{x}_{\Gamma}\right)$.

- If $m$ comes from finite difference data such as $G$, we construct a trilinear approximation using the nodes of the element containing $\mathbf{x}_{\Gamma}$.

Since we use trilinear finite elements, we use this approximation order also in the finite difference discretization. However, we note that for evaluating $f\left(\mathrm{x}_{\Gamma}\right)(2.11)$ we combine other types of representations: $\mathbf{u}$ and $E_{n}$ involve gradients of finite element functions and $\kappa(5.5)$ is a tricubic function. Regardless of representation, the initial data is an evaluation of the function in the closest points. After computing $m$ on the front, we use the extension velocity procedure as described in [31] using a heap structure to compute $m_{\text {ext }}$. 


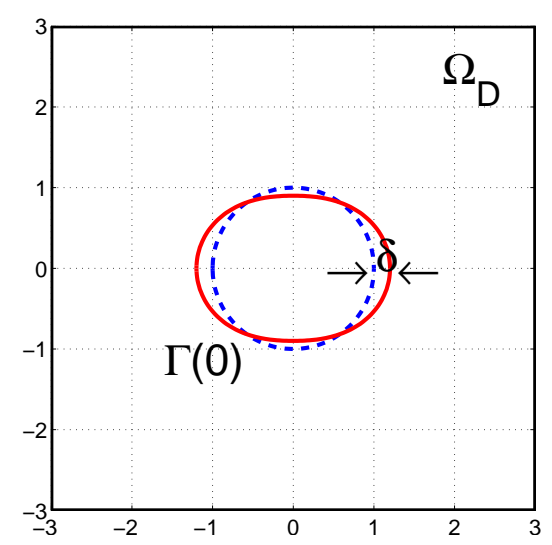

Figure 6.1: Initial front for the oscillating sphere.

\section{Numerical tests for the fluid dynamics problem}

In this section we first present the standard convergence analysis with respect the discretization parameters, using the linear solution of the oscillation sphere [34. To test the behavior of the fully 3D algorithm when the fluid undergoes topological change, a two lobe drop geometry, which is known to pinch-off, is taken as the initial front. Both examples have served as case studies to carefully validate the boundary integrallevel set numerical method used to approximate the axi-symmetric version of Eqns. 2.16) to (2.20) before and after pinch-off, see [12, 13. The full 3D results will be compared with the corresponding axi-symmetric computations when needed.

\subsection{Examination of Nitsche methods: The oscillating sphere}

Assuming rotational symmetry around the $z$ axis, if a unit sphere is initially perturbed as

$$
\begin{aligned}
& z(s)=-\cos (s)\left(1+\delta P_{m}(\cos (s))\right) \\
& r(s)=\sin (s)\left(1+\delta P_{m}(\cos (s))\right),
\end{aligned}
$$

where $0 \leq s \leq \pi$ and $P_{m}$ the Legendre polynomial of order $m \in \mathbb{N}$ (see Fig. 6.1), the sphere then oscillates with frequency

$$
\omega^{2}=\frac{m(m-1)(m+2)}{m+1}
$$

for small $\delta$. Here $r=\sqrt{x^{2}+y^{2}}$ and $z$ is the symmetry axial coordinate. To obtain the initial front for the $3 \mathrm{D}$ computations, the axi-symmetric profile has been rotated around the $z$ axis, $\Gamma(0)=\{(x, y, z) \mid x=$ $r(s) \cos (\theta), y=r(s) \sin (\theta), z=z(s), 0 \leq \theta<2 \pi\}$ and the initial velocity potential set to zero.

The fixed computational domains is $\Omega_{D}=[-3,3]^{3}$. The perturbation is set to $\delta=0.2$ and $m=2$ gives an oscillation period of $T_{e}=2.2214$. The value calculated using the axi-symmetric approach is $T_{c}=2.288$ [13.

\subsubsection{Testing the non-symmetric and symmetric Nitsche methods}

We will now test the non-symmetric $(\sqrt{4.9}, \sqrt{4.10})$ ) and symmetric $(44.7),(4.8))$ methods with both the ghost penalty 4.15 and the gradient jump 4.30 stabilization terms. The tests are run on a mesh with $N=51$ points in each direction and $\Delta t=0.001$. The left graph of Fig. 6.2 shows the kinetic energy as a function of time for the non-symmetric method using the ghost penalty stabilization for a number of values of the $\gamma_{g p}$ parameter. The right graph show the corresponding results using the gradient jump stabilization. The results show that using the ghost penalty stabilization, smoother energies are obtained compared to 

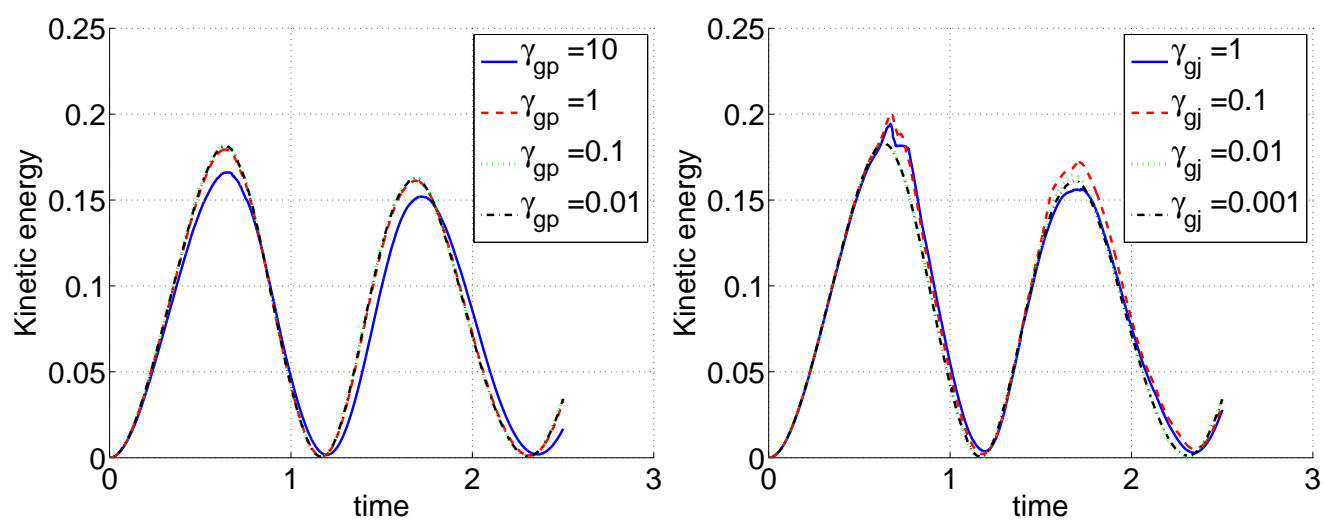

Figure 6.2: The kinetic energy for the non-symmetric method with ghost penalty stabilization (left) and gradient jump stabilization (right) for different values of the stabilization parameters.

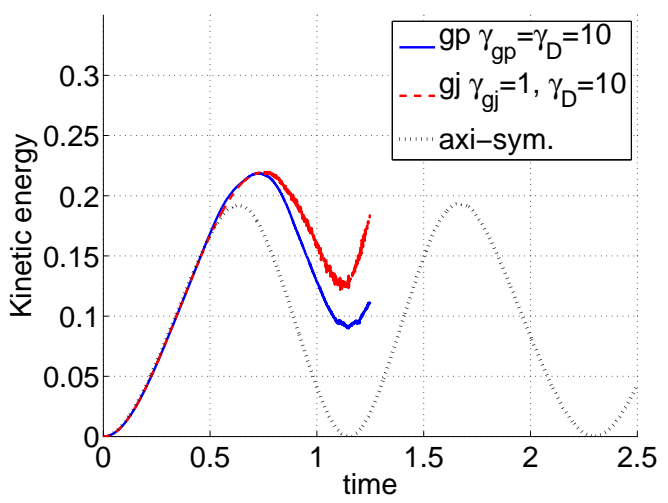

Figure 6.3: The kinetic energy for the symmetric method with ghost stabilization ("gp") and gradient jump stabilization ("gj"). For reference, the dash-dotted line is the kinetic energy for the non-symmetric method and the dotted line is the kinetic energy from the axi-symmetric code [13].

the gradient jump stabilization. On the other hand, the energies are more damped when using the ghost penalty stabilization compared to that of the gradient jump stabilization. However, experience has shown that it is important to obtain smooth velocity fields, and thus the ghost penalty stabilization with $\gamma_{g p}<10$ is preferred.

The symmetric method performs poorly regardless of stabilization for all parameter values tested, namely $\gamma_{i}=\{0.01,0.1,1,10,100\}, i \in\{g p, g j, D\}$. For many parameter combinations, the linear system fails to converge. At best, too large and/or wiggly kinetic energies are obtained, as illustrated in the ghost penalty stabilization example in Fig. 6.3. Other parameter combinations yield sharp increases in kinetic energy, such as the results using the $S_{\text {opt }}$ parameter set in Fig. 6.3. We did not continue the simulations since the results were clearly inadequate.

To this end we conclude that the only viable choice for this problem is the non-symmetric method with ghost penalty stabilization.

\subsubsection{Convergence tests using the non-symmetric method with ghost penalty stabilization}

Below we show the results of running for two complete oscillation periods with $N$ number of points in each mesh direction, thus the mesh size is uniform $\Delta x=\Delta y=\Delta z=h$. Simulations were performed for the 


\begin{tabular}{|crccc|}
\hline case & \multicolumn{1}{c}{$N$} & $T_{c}$ & $e_{V}$ & $e_{E}$ \\
\hline (a) & 51 & 2.308 & 0.0468 & 0.0440 \\
(b) & 101 & 2.292 & 0.0267 & 0.0232 \\
(c) & 201 & 2.291 & 0.0179 & 0.0123 \\
\hline
\end{tabular}

Table 6.1: Oscillation period, relative error in volume and in total energy at $t=2.5$.

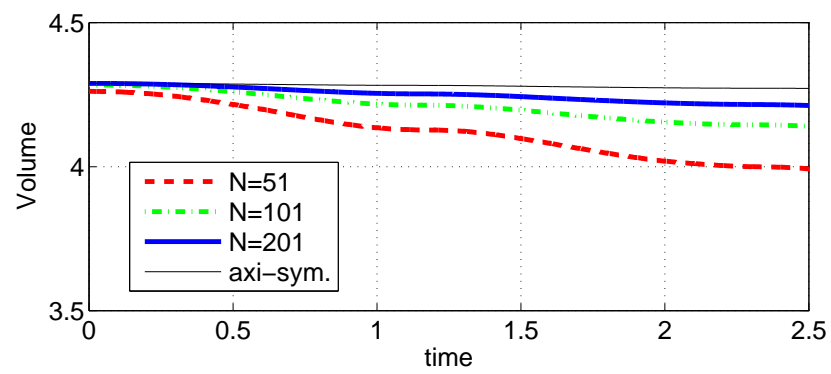

Figure 6.4: Volume evolution for the oscillating sphere.

following sets of discretization parameters using $\gamma_{g p}=1$
(a) $\quad N=51, h=0.12, \Delta t=0.001$
(b) $N=101, h=0.06, \Delta t=0.001$
(c) $\quad N=201, h=0.03, \Delta t=0.0005$

Fig. 6.4 and 6.5 show the drop volume and kinetic energy evolution respectively for the different meshes and in Fig. 6.6 the evolution of points $(x(t), 0,0)$ and $(0,0, z(t))$ is also displayed. The results corresponding to the axi-symmetric computation are also shown in each of these figures for comparison.

Table 6.1 lists the values of the calculated oscillation period $T_{c}$, the relative error in drop volume $e_{V}$, and the relative error in total energy $e_{E}$, at $t=2.5$ for each case. We can conclude that first order convergence in space is achieved (as expected) and the results obtained with the full $3 \mathrm{D}$ algorithm coincide with those given by the axi-symmetric method.

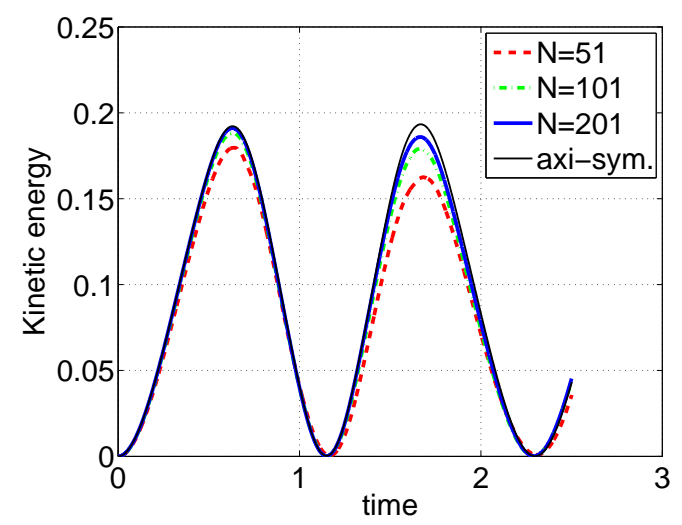

Figure 6.5: Kinetic energy evolution for the oscillating sphere. 


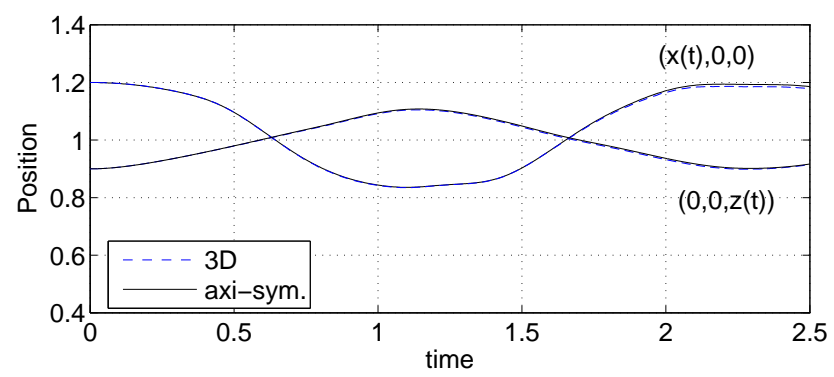

Figure 6.6: Points $(x(t), 0,0)$ and $(0,0, z(t))$ evolution for the oscillating sphere.

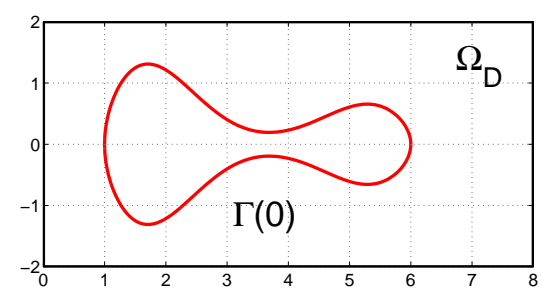

Figure 6.7: Initial front for the two lobe geometry drop.

\subsection{Investigating topological change: Droplet break-up}

One advantage with the level set method is that it is straightforward to handle topological changes of the free boundary. To study topology changes in the setting of Nitsche's method we take as reference problem the two lobe geometry from [34, which is known to pinch-off. Note that to initialize the system (3.1) (3.4), the front position $\Gamma(0)=(x(0), y(0), z(0))$ and the velocity potential $\left.\phi\right|_{\Gamma(0)}$ are needed. We take the $2 \mathrm{D}$ axi-symmetric geometry as in 34$]^{1}$ which was used to test the axi-symmetric algorithm in [13]:

$$
\begin{aligned}
& z(s)=1+B(1-a(s)) \\
& r(s)=g_{1}(s)+g_{2}(s) \\
& a(s)=(\cos (s)+1) / 2
\end{aligned}
$$

where

$$
\begin{aligned}
& g_{1}(s)=\sqrt{B} \sqrt{a(s)}\left(\mathrm{e}^{-B^{2}(a(s))^{2} / 2}-\mathrm{e}^{-B^{2} / 2}\right) \\
& g_{2}(s)=C \sqrt{B} \sqrt{1-a(s)}\left(\mathrm{e}^{-B^{2}(a(s)-1)^{2} / 2}-\mathrm{e}^{-B^{2} / 2}\right),
\end{aligned}
$$

and $0<s<\pi$. The initial geometry using $B=5$ and $C=2$ is depicted in Fig. 6.7. The 3D initial front is obtained rotating the $2 \mathrm{D}$ data, we set $\left.\phi\right|_{\Gamma(0)}=0$ and $\Omega_{D}=[-2,2]^{2} \times[0,8]$.

The number of points taken for this computation are $N=M=129$ and $L=257$, which give a uniform grid spacing of $h=0.03125$. The time step is fixed along the calculation to $\Delta t=0.001$. Note that we have a unique spatial discretization parameter $h$. Moreover, the fact that no explicit reconstruction and discretization of the free surface is needed (as in the boundary element method) makes the computation simpler, particularly when the initial domain splits in several disconnected domains.

To validate the 3D computed profiles we compare drop shapes and time occurrences with the axisymmetric results. Here, we are assuming that the axi-symmetric computation is reliable, as all the available tests, including near pinch-off self similar solutions, have already been performed, see [14]. In Fig. 6.8 we

\footnotetext{
${ }^{1}$ The formulas were kindly supplied by Professor David Leppinen
} 


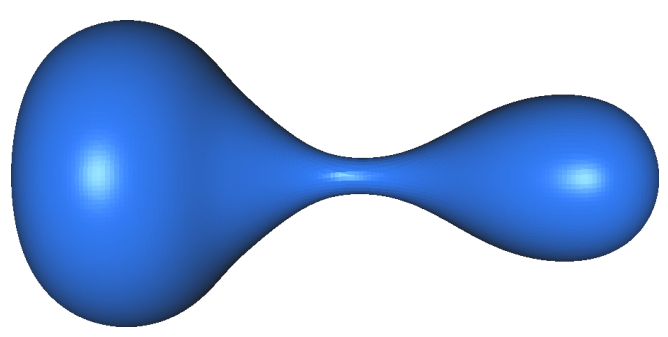

(a) $t=0.235$

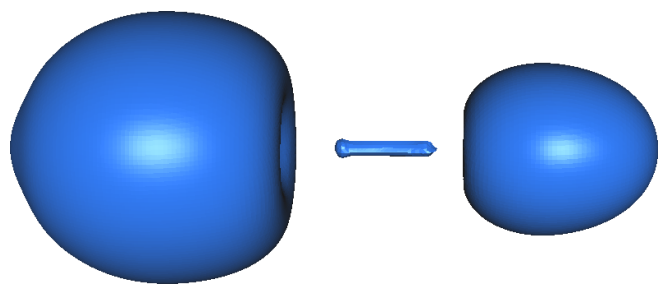

(c) $t=0.520$

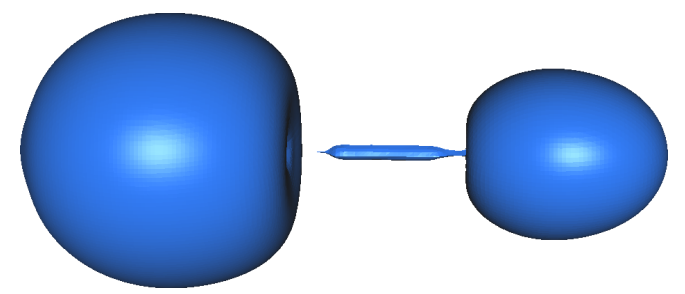

(b) $t=0.495$

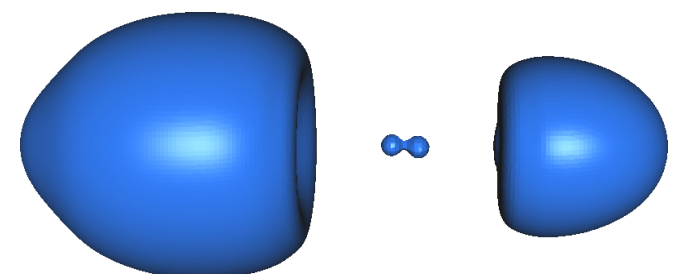

(d) $t=0.600$

Figure 6.8: Evolution of the two lobe drop.

show the three-dimensional drop at four different times, and in Fig. 6.9 we show a comparison with the axi-symmetric profiles from [13, and also show the velocity potential at a cross section in the center of the drop. The largest differences appear close to pinch-off time, and appear later using the method presented in this paper. The reason for this may be the coarser mesh used here, which is not able to capture the large curvatures to the same extent as in the axi-symmetric computation, which uses a finer discretization.

Regarding the physical interpretation of the results, the algorithm presented makes it possible to extract drop profiles as the zero iso-surface of the level set function. The complete flow pattern inside the droplet is also provided. This gives additional and valuable information when compared to previous approaches, since velocity potential iso-contours and $3 \mathrm{D}$ velocity fields are missing (or very costly to recover) in a boundary integral approach. Although these variables can be visualized in 3D, we choose to show, as in Figs. 6.9, 6.10 and 6.11, cross sections through the center of the drop.

At $t=0.445$ a strong suction effect towards the left fluid mass is taking place, as show by the magnitude of the velocity arrows in Fig. 6.10 bottom. At the same time, in Fig. 6.10 top, we display velocity arrows without scaling to show how the flow separates in the center of the left fluid mass. The fluid coming from the drop center to the right faces the incoming flow from the neck creating a preference flow direction (approximately 45 degrees from the central drop axis), which will give rise to the drop profile overturning before pinching.

At $t=0.600$ (see Fig. 6.11), after the second pinch-off event, the satellite drop undergoes a fast contraction from filament shape to two lobe shape. The left drop is expanding to the left and the right drop is still contracting towards its center. The surface points in both main drops exhibiting an outgoing velocity profile normal to the surface indicate the formation of capillary waves that will propagate through the liquid surface, creating drop oscillating shapes until an equilibrium geometry is reached.

We point out that the velocity vectors in the drop axis throughout the whole simulation are horizontal, indicating that the $3 \mathrm{D}$ computation is preserving the axi-symmetric character of this particular flow.

\section{Electrohydrodynamic simulations}

The dynamics of drops and films change remarkably when the liquid is subject to electric forces. As numerous experiments in electrospraying show, when the electric field intensity is sufficiently strong thin fluid jets are ejected from conical tips formed in the fluid surface [5, 35]. 

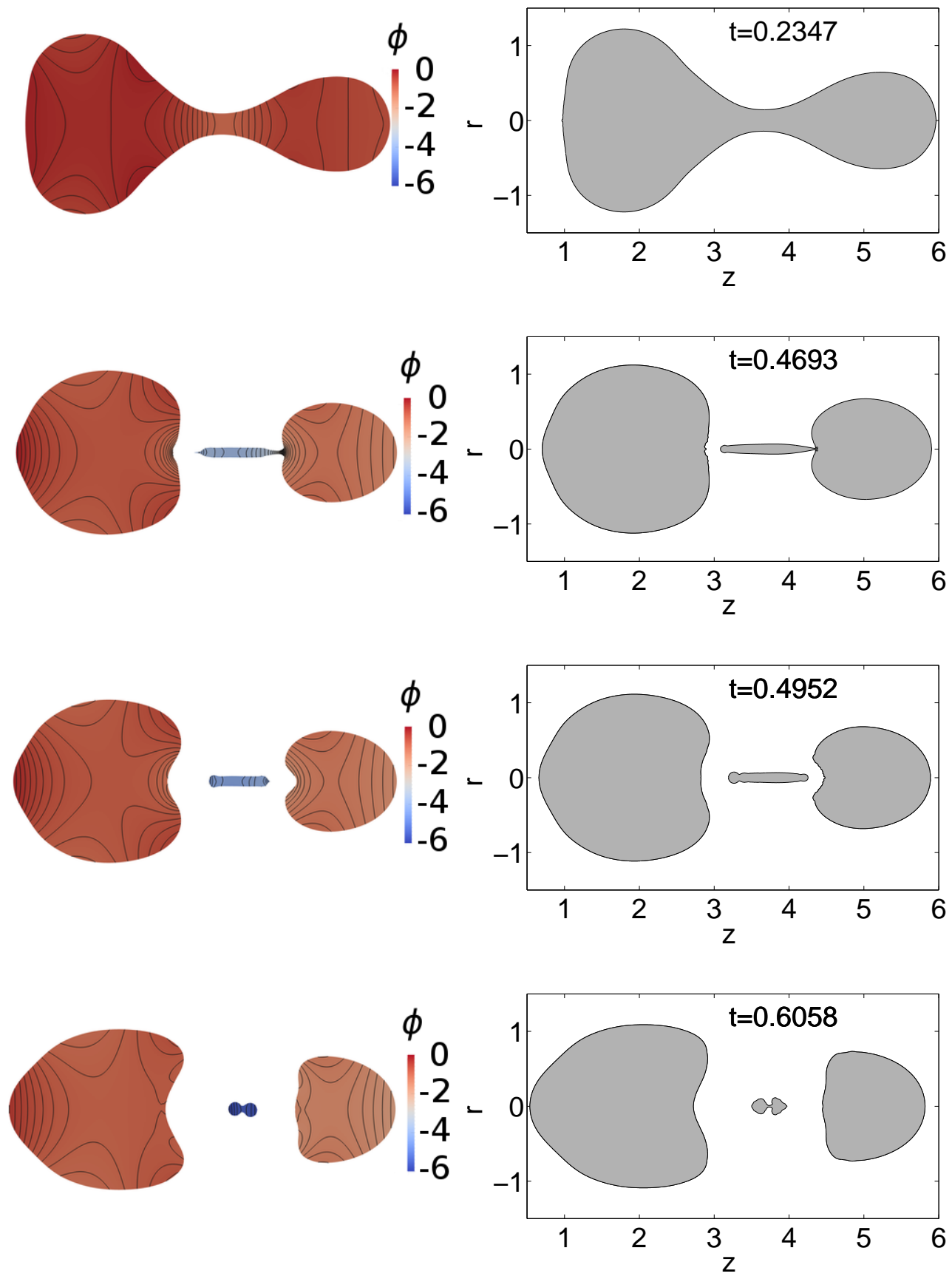

Figure 6.9: Comparison of axi-symmetric and full 3D computation. Left column show a cross section of the drop showing the velocity potential. Right column is the axi-symmetric reference computation. The times for the 3D computations are from top to bottom: $0.235,0.495,0.520$ and 0.600 . There is a slight difference in pinch-off time. 

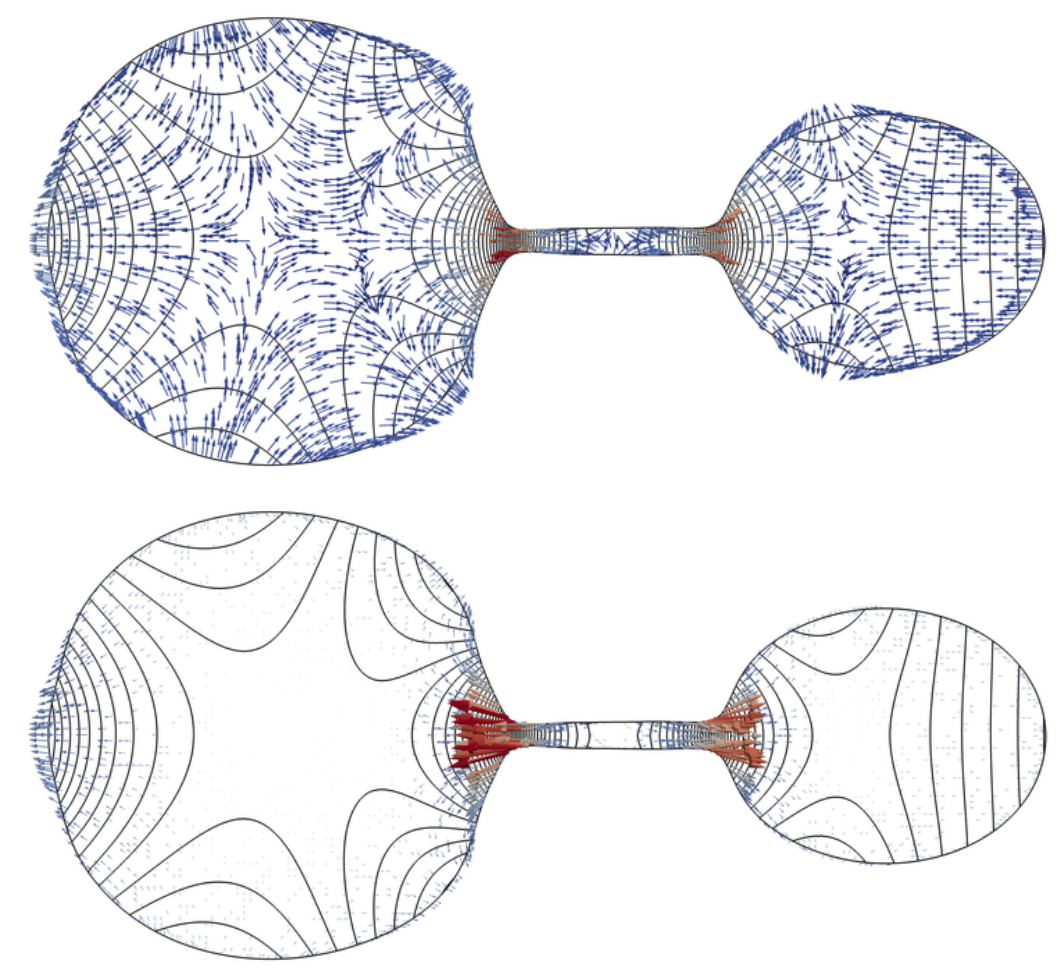

Figure 6.10: Velocity field at $t=0.445$ illustrated by fixed and speed-proportional arrows. The flow does not look perfectly symmetric since the visualizer (Paraview) uses random points to draw the arrows.
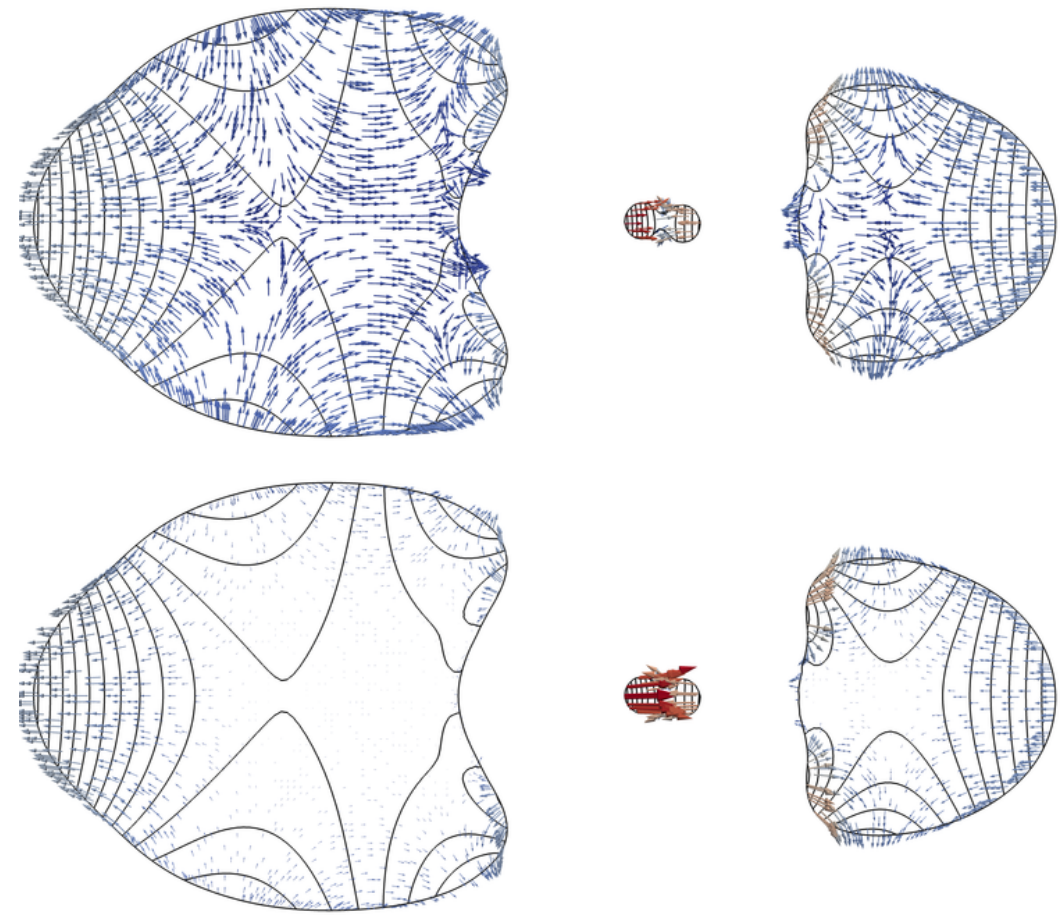

Figure 6.11: Velocity field at $t=0.600$ illustrated by fixed and speed-proportional arrows. The flow does not look perfectly symmetric since the visualizer (Paraview) uses random points to draw the arrows. 


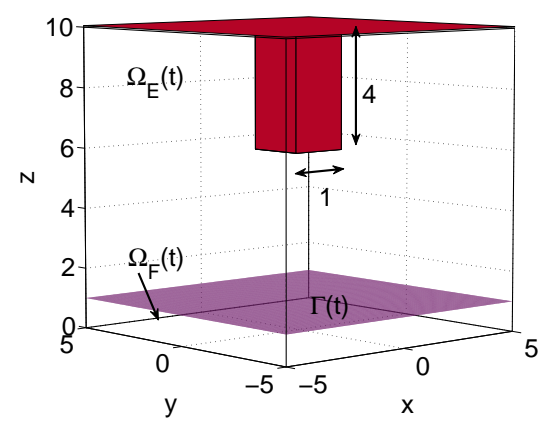

Figure 7.1: Experimental setup of 44].

For neutral non-viscous droplets, by assuming the evolution shapes were prolate spheroids, Taylor (see [8, 9]) found a critical electric field intensity above which the droplet becomes unstable. His theory predicts the formation of a perfect (Taylor) cone and symmetric jet discharge follows at both ends of the elongated droplet. This case, for neutral and charged droplets, viscous and inviscid liquids, has given rise to various theoretical, see 36 38, numerical [14, 39,41] and experimental studies [42, 43.

Although the ultimate goal is the numerical simulation of an electrospray flowing from a nozzle at different flow rates and electric field strengths, in this paper we start with a simpler case study, namely the electrohydrodynamic deformation and induced tip streaming from a thin non-viscous fluid film. Following the work by Collins et al. [44, we start with the same physical setting as the one shown in figure $1 a$ of their paper. As we do not have the axi-symmetric restriction, we take a rectangular box. At the bottom and top faces two squared electrodes are placed forming a parallel plate capacitor. Protruding from the center of the top electrode there is a prismatic metal bar, see Fig. 7.1

A perfectly conducting non-viscous liquid film with constant height $z_{0}$, sits over the bottom horizontal metallic plate while the rest of the box is filled with an insulating gas. A constant potential difference $V_{0}$ is set between the two parallel electrodes which, due to electrical stresses $E_{n}=\mathbf{n} \cdot \nabla V$ acting on the free boundary, induce an upward displacement of the fluid surface. Let $\Omega_{F}(t)$ and $\Omega_{E}(t)$ be the fluid and insulating gas domains respectively and $\Gamma(t)$ the moving surface between them, considering the assumptions exposed in Section 2, the Eulerian model equations, Eqns. 2.16) to (2.23), apply for this case. Now, in addition to solving a Laplace equation for the fluid velocity potential in $\Omega_{F}(t)$, we have to solve an additional equation to obtain the electric potential in $\Omega_{E}(t)$. The semi-discrete system for the electrohydrodynamic problem is given by

$$
\begin{aligned}
\mathbf{u}^{n} & =-\nabla \phi^{n} \quad \text { in } \Omega_{F}\left(t_{n}\right) \\
\Delta \phi^{n}(x, y, z) & =0 \quad \text { in } \Omega_{F}\left(t_{n}\right) \\
\left.\phi^{n}\right|_{\Gamma_{t_{n}}} & =\left.G^{n}\right|_{\Gamma_{t_{n}}} \\
\frac{\Psi^{n+1}-\Psi^{n}}{\Delta t} & =-\mathbf{u}_{\text {ext }}^{n} \cdot \nabla \Psi^{n} \quad \text { in } \Omega_{D} \\
\frac{G^{n+1}-G^{n}}{\Delta t} & =-\mathbf{u}_{\text {ext }}^{n} \cdot \nabla G^{n}+f_{\text {ext }}^{n} \quad \text { in } \Omega_{D} . \\
\mathbf{E}^{n} & =-\nabla V^{n} \quad \text { in } \Omega_{E}\left(t_{n}\right) \\
\Delta V^{n}(x, y, z) & =0 \quad \text { in } \Omega_{E}\left(t_{n}\right) \\
\left.V^{n}\right|_{\Gamma_{t_{n}}} & =0
\end{aligned}
$$

The boundary conditions for the lateral box faces of the narrow band are $\mathbf{n} \cdot \nabla \Psi=0$ for Eqn. (7.4) and $\mathbf{n} \cdot \nabla G=0$ for Eqn. 7.5 . The boundary conditions for Eqn. 7.2 at the fixed boundaries of $\Omega_{F}(t)$ are $\mathbf{n} \cdot \nabla \phi=0$. Similarly we set $\mathbf{n} \cdot \nabla V=0$ at the four lateral faces enclosing $\Omega_{E}(t)$ and $V=1$ at the top electrode for Eqn. (7.7). 
The non-symmetric Nitsche method, as detailed in Section 4 , is used to obtain the surface gradients $E_{n}$ on the free surface. These are needed to compute $f_{\text {ext }}^{n}$ of Eqn. 7.5). As in Section 6, we use the ghost penalty stabilization (4.15) and here we let $\gamma_{g p}=4$. To make the steps clear we briefly describe the algorithm in Alg. 7.1 .

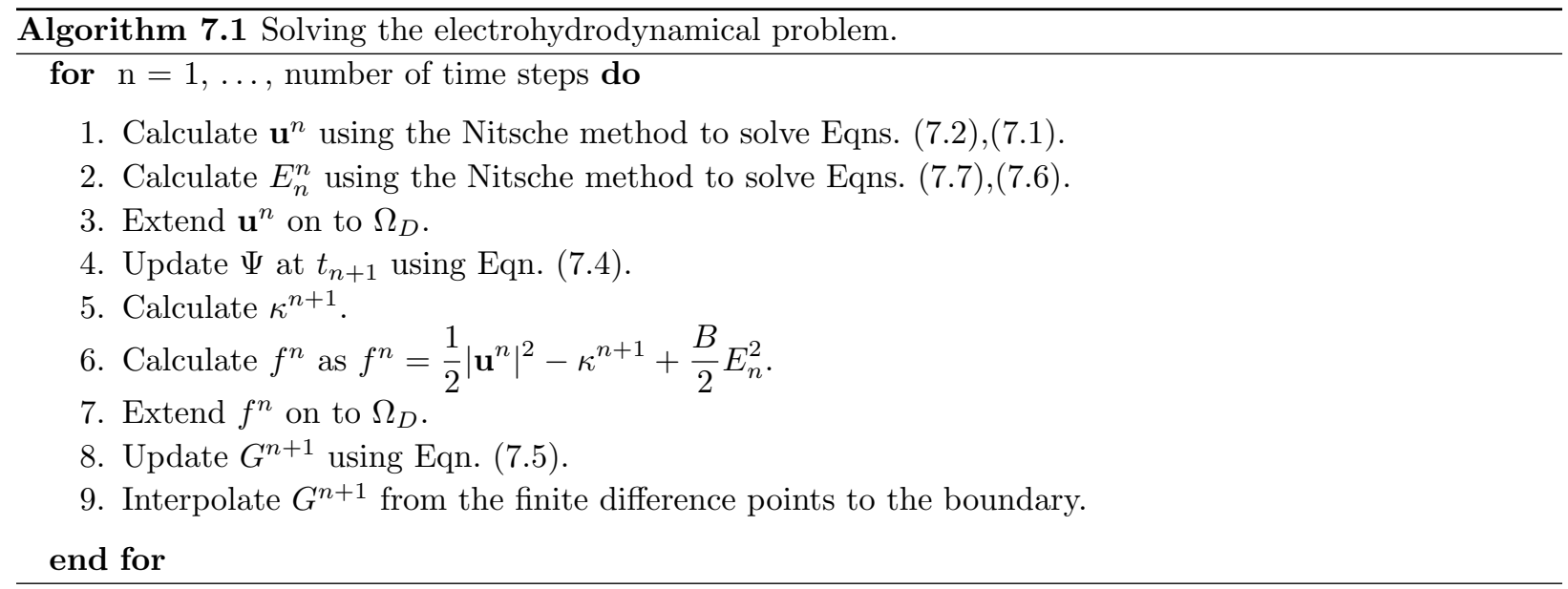

\subsection{Numerical results}

As mentioned above, we follow [44] and consider a box of dimensions $[-5,5]^{2} \times[0,10]$ with a protruding bar in the top electrode with dimensions $[-1,1]^{2} \times[6,10]$ as shown in Fig. 7.1. The computational domain $\Omega_{D}=[-5,5]^{2} \times[0,10]$ is the physical domain between the two electrodes. We set the initial position of the liquid film at $z_{0}=1$. To investigate the mesh dependencies, the number of points is chosen to be $N=41$, 81 and 161 which corresponds to grid spacings of $h=0.25,0.125$ and 0.0625 . The time step used is set to depend on the maximum speed $|\mathbf{u}|$ by letting $\Delta t=0.01$ in the beginning and decreased to $\Delta t=0.0005$ at the time of jetting.

To study the behavior of the system with respect the Bond number $B$, we have run simulations with $B \in\{20,30,40\}$. Above a critical Bond number $B_{c}$, conical tip streaming will take place. In Fig. 7.2 we show $3 \mathrm{D}$ profiles of the water surface and the iso-surfaces of the electric potential for various times using $B=40$. In the same figure we also show the velocity field of the fluid.

In Fig. 7.3 we plot the aspect ratio $z_{\max } / z_{0}$ evolution for the three Bond numbers and the three mesh sizes. For this problem $z_{\max }$ will occur along the $z$-axis at $x=y=0$. As can be seen, $B_{c} \in(20,30)$ since we do not get tip formation for $B=20$, whereas we indeed obtain a tip for $B=30$ and 40 .

Running with $B=20$ over long time we obtain an oscillatory behavior as illustrated in Fig. 7.4. The reason for this is the balance of capillary and electrical forces: At time zero, the capillary forces are zero and there are only electrical forces. During evolution, $z_{\max }$ keeps increasing until the capillary forces overcome the electric forces, causing the reversal movement. This process repeats itself with a period that will depend on the Bond number. In 14, this phenomenon is also observed in the case of an isolated droplet. Due to the extensive computational time, this simulation was only performed on the coarsest mesh $N=41$.

In Fig. 7.5 we show velocities and electric stresses for $B=30$ and 40 and the three mesh sizes. We observe that for the higher Bond number, the variables diverge earlier as expected. We only provide data until the time of singularity, at approximately $t=12$ for $B=30$ and $t=8$ for $B=40$. At this time, the Taylor cone is created and thin jets are formed giving rise to a cone bursting mode.

Using $B=30$ or $B=40$, jetting of a small drops is observed from approximately $t=12$ and $t=8 \mathrm{~s}$, respectively. This is illustrated in Fig. 7.6 for $B=40$. Regarding the physical interpretation of Fig. 7.6, the authors believe that the spatial scale of $N=161$ is not sufficiently fine to capture the accurate shape of the cone. With $N=161$, the width of the jet and the ejected drop is only two to three elements wide. 

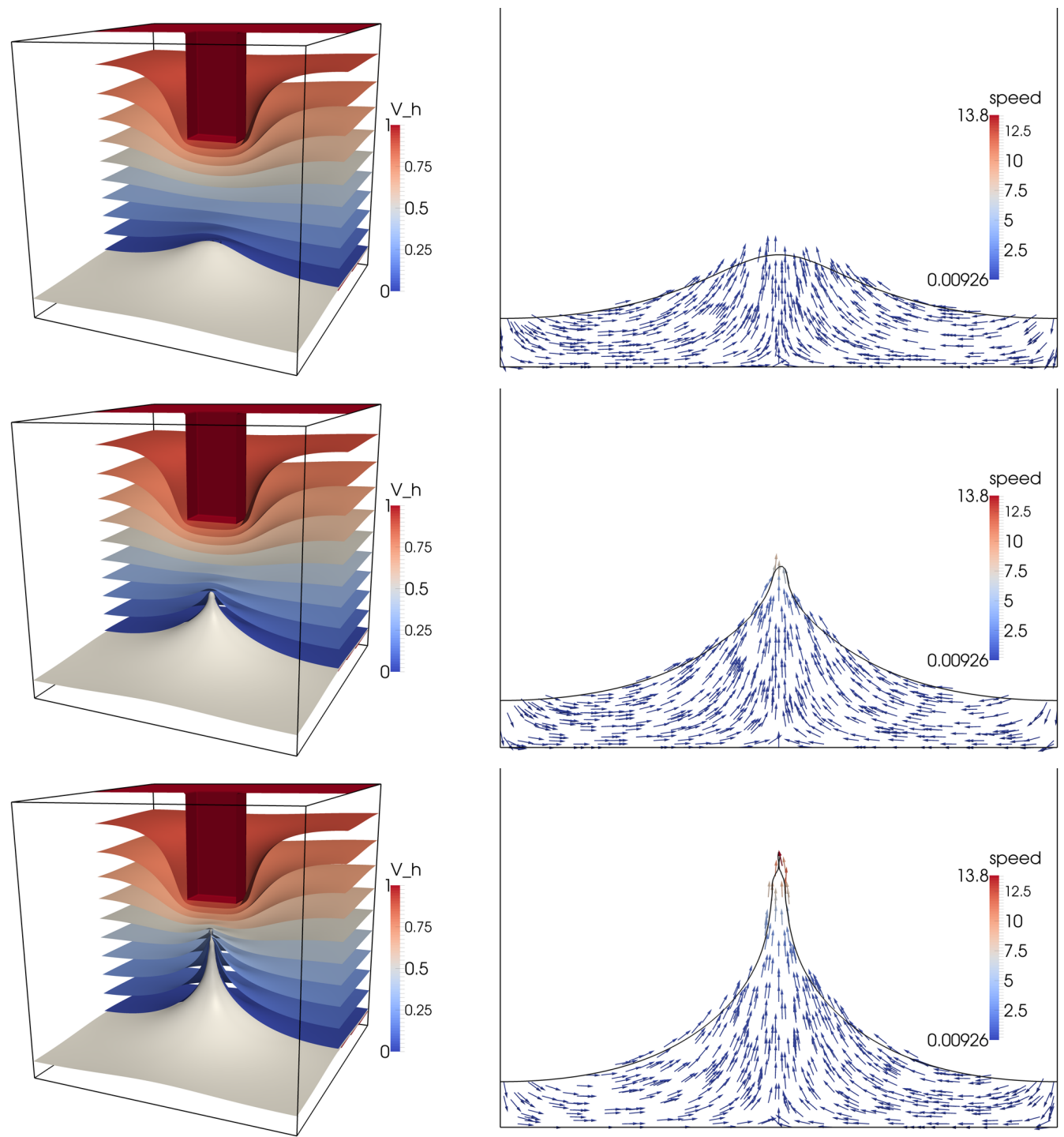

Figure 7.2: Iso-surfaces of the electric potential $V_{h}$ (left) and velocity field (right) for $B=40$ and $t=7.25,7.95$ and 8.5625 . Since the velocity field is plotted on $\mathcal{K}_{F}(t)$ and the glyphs are plotted at random mesh vertices, some glyphs are outside $\Gamma(t)$.
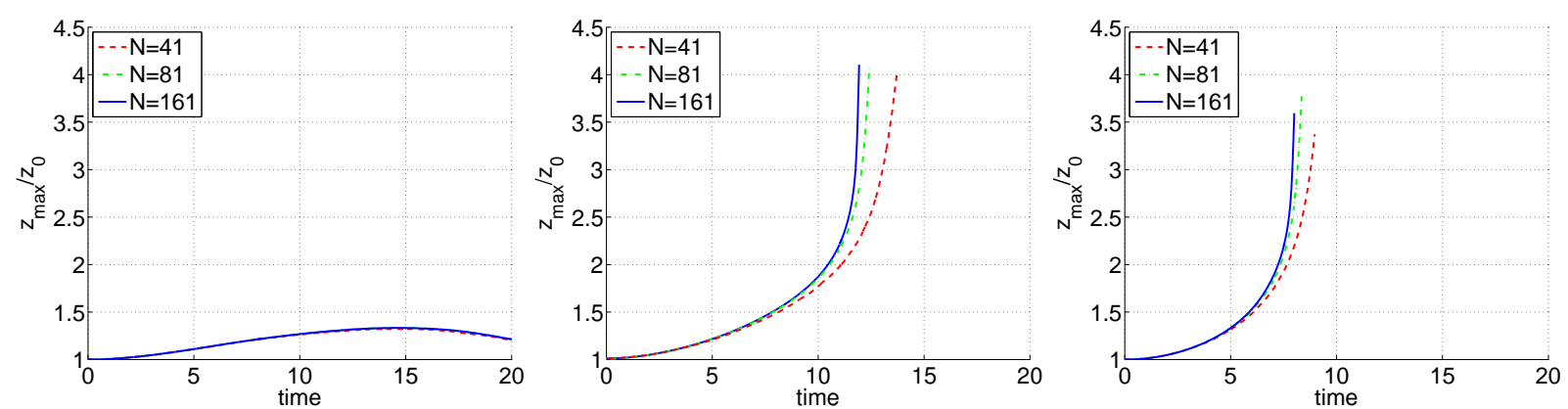

Figure 7.3: The aspect ratio $z_{\max } / z_{0}$ as a function of time for $B=20,30$ and 40 (left to right). 


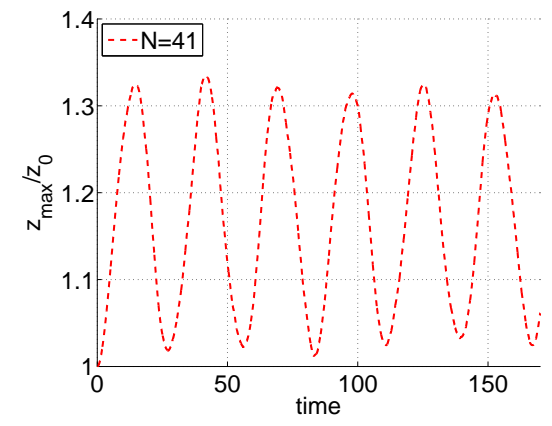

Figure 7.4: Oscillatory behavior when $B=20$.
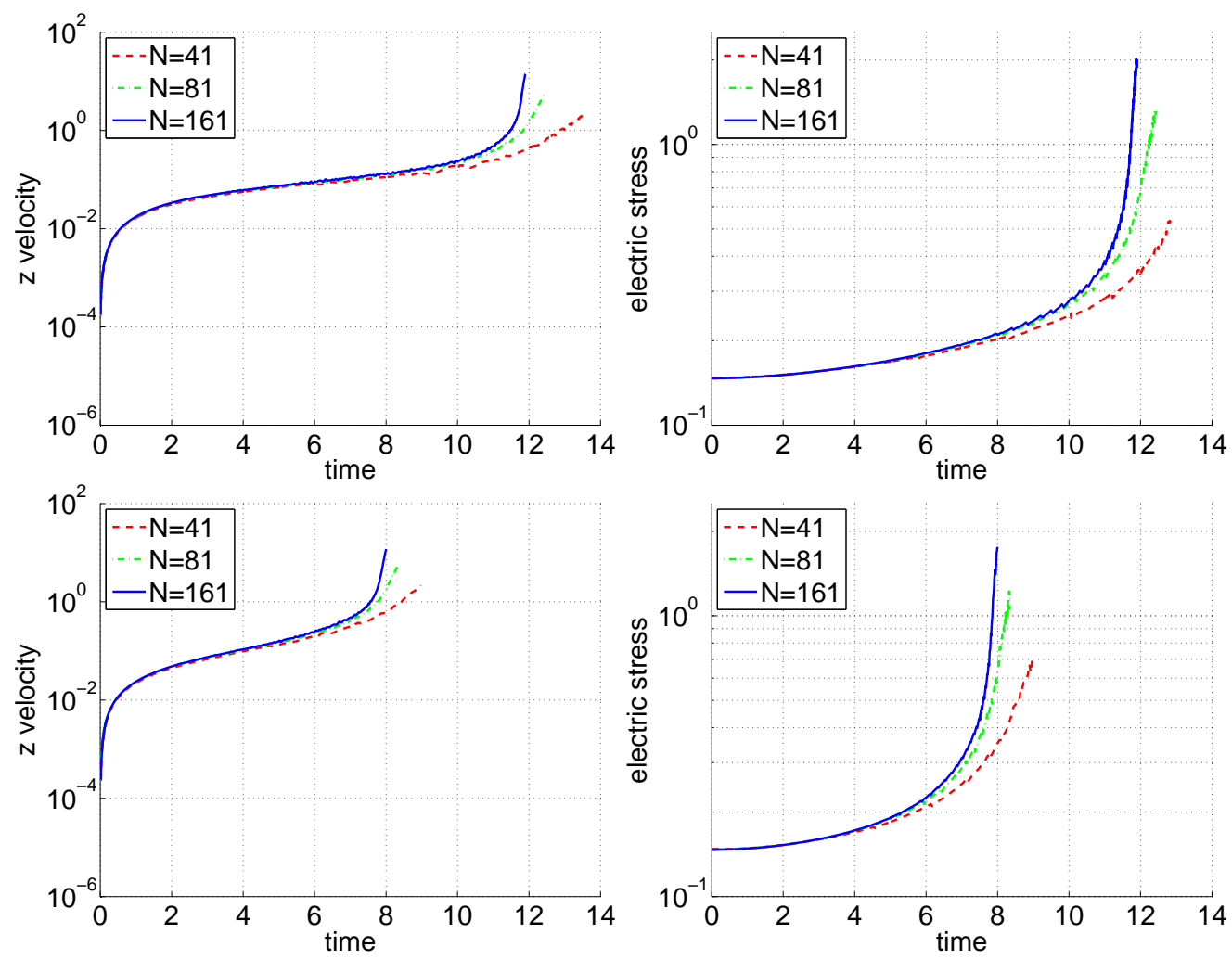

Figure 7.5: The velocity and electric stress at $z_{\max }$ using $B=30$ (top row) and $B=40$ (bottom row). 

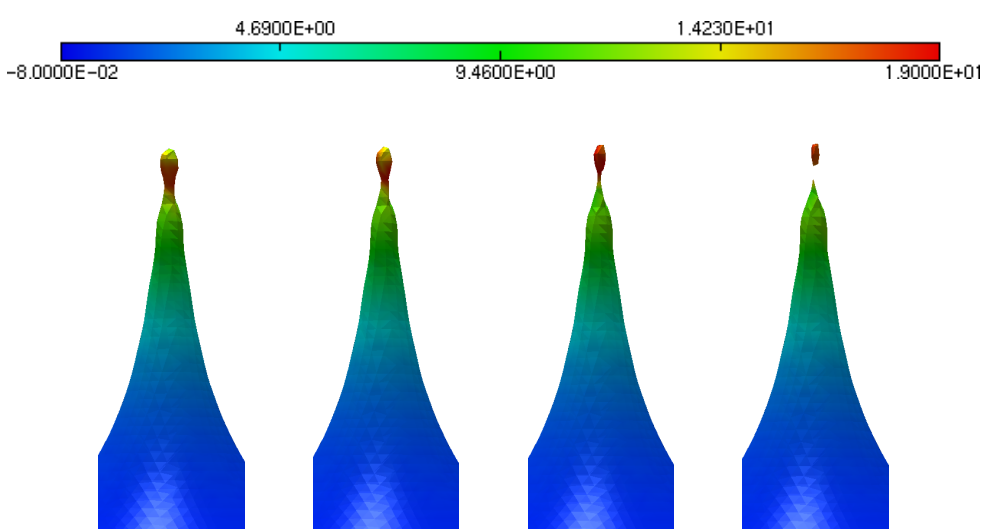

Figure 7.6: Zoom of the tip at times $t=8.5575,8.56,8.5625$ and 8.565 (left to right) with $B=40$. Colors represent velocities in the $z$ direction.

Using $N=161$, each time step took about $140 \mathrm{~s}$ on a standard desktop computer (as of 2014) with an intel i7 cpu. The number of unknowns varies in each time step, but towards jetting it was approximately 460000 for the fluid potential and 3.7 million for the electric potential. Total simulation time for the longest simulation was about 5 days.

\section{Conclusions}

In this paper we have developed a three-dimensional algorithm, based on the Nitsche-level set coupling to approximate hydrodynamic problems with free boundaries. Using the level set/extended potential model, singular flow events, such as fluid break-up or merging, are easily handled and the computations can go past these singular times.

We have investigated the performance of the Nitsche finite element method in the context of timedependent problems and with respect to computing the gradients on the free boundary, since these are input to level set equations modeling the dynamics. In particular, the symmetric and non-symmetric forms of the Nitsche method and two stabilization techniques have been studied. Numerical test for static and dynamic problems have been selected to review these methods. The non-symmetric Nitsche method combined with the ghost penalty stabilization turned out to be the preferred option for the type of problems considered here.

Several numerical test to validate the algorithm have been presented: The oscillation of a perturbed sphere was used to establish the computational convergence of the method; The break-up of a two lobe axi-symmetric droplet has not only shown the capability of the algorithm to handle topological changes but also to preserve axi-symmetric flow patterns. Moreover, the numerical results, drop profiles and time events, are in very good agreement with previous axi-symmetric calculations.

The deformation and tip streaming of a thin fluid film under the action of electrical forces has been numerically studied. To include the effect of electric stresses on the fluid motion an exterior Laplace problem corresponding to the electric potential has to be approximated. The onset of tip streaming depends upon the intensity of the electric field applied, which is represented by the electric Bond number. We present numerical results for various Bond numbers. Above the critical value our simulations show the formation of a thin jet and the subsequent drop ejection.

Performing full 3D simulations is very costly. In future work, the authors plan to investigate adaptive meshes which seem crucial to capture the small features involved in the jetting process. 


\section{Acknowledgments}

This work was supported in part by the Applied Mathematical Science subprogram of the Office of Energy Research, U.S. Department of Energy, under contract Number DE-AC02-05CH11231. The first author was also supported by The Research Council of Norway through a Centres of Excellence grant to the Center for Biomedical Computing at Simula Research Laboratory, Project Number 179578. The second author was also supported by the Spanish Ministry of Science and Innovation, Project Number MTM2013-43671-P. The third author was also supported by the Division of Mathematical Sciences of the U.S. National Science Foundation, and acknowledges the support as an Einstein Visiting Fellow of the Einstein Foundation, Berlin, Germany.

[1] E. Schaffer, T. Thurn-Albrecht, T. P. Russell, U. Steiner, Electrically induced structure formation and pattern transfer, Nature 403 (2000) 874877. doi:10.1038/35002540

[2] N. Snook, M. Xue, Effects of microphysical drop size distribution on tornadogenesis in supercell thunderstorms, Geophysical Research Letters 35 (24). doi:10.1029/2008GL035866

[3] J. D. Yu, S. Sakai, J. A. Sethian, A coupled level set-projection methods applied to ink jet simulations, Interfaces and Free Boundaries 5 (2003) 459-482. doi:10.4171/IFB/87.

[4] S. Nudurupati, M. Janjua, P. Singh, N. Aubry, Electrohydrodynamic removal of particles from drop surfaces, Phys. Rev. E 80 (2009) 010402. doi:10.1103/PhysRevE.80.010402

[5] I. Marginean, P. Nemes, A. Vertes, Astable regime in electrosprays, Phys. Rev. E 76 (2007) 026320. doi:10.1103/ PhysRevE.76.026320

[6] W. D. Ristenpart, J. C. Bird, A. Belmonte, F. Dollar, H. A. Stone, Non-coalescence of oppositely charged drops, Nature 461 (2009) 377-380. doi:10.1038/nature08294

[7] Lord Rayleigh, On the equilibrium of liquid conducting masses charged with electricity, Philosophical Magazine 14 (1882) 184-186. doi:10.1080/14786448208628425

[8] G. Taylor, Disintegration of water droplets in an electric field, P. Roy. Soc. Lond. A. (1964) 383-397doi:10.1098/rspa. 1964.0151

[9] G. Taylor, Electrically driven jets, P. Roy. Soc. Lond. A. 313 (1969) 453-475. doi:10.1098/rspa.1969.0205

[10] M. Garzon, D. Adalsteinsson, L. J. Gray, J. A. Sethian, A coupled level set-boundary integral method for moving boundary simulations, Interfaces and Free Boundaries 7 (2005) 277-302. doi:10.4171/IFB/125.

[11] M. Garzon, L. J. Gray, J. A. Sethian, Numerical simulation of non-viscous liquid pinch-off using a coupled levelset-boundary integral method, J. Comput. Phys. 228 (2009) 6079-6106. doi:10.1016/j.jcp.2009.04.048

[12] M. Garzon, L. J. Gray, J. A. Sethian, Simulation of the droplet-to-bubble transition in a two-fluid system, Phys. Rev. E 83 (2011) 046318. doi:10.1103/PhysRevE.83.046318.

[13] M. Garzon, L. J. Gray, J. A. Sethian, Axisymmetric boundary integral formulation for a two-fluid system, Int. J. Numer. Meth. in Fluids 69 (2012) 1124-1134. doi:10.1002/fld.2633

[14] M. Garzon, L. J. Gray, J. A. Sethian, Numerical simulations of electrostatically driven jets from nonviscous droplets, Phys. Rev. E 89 (2014) 033011. doi:10.1103/PhysRevE.89.033011.

[15] J. Nitsche, Über ein Variationsprinzip zur Lösung von Dirichlet-Problemen bei Verwendung von Teilräumen, die keinen Randbedingungen unterworfen sind, Abh. Math. Sem. Hamburg 36 (1971) 9-15. doi:10.1007/BF02995904

[16] E. Burman, Ghost penalty, Comptes Rendus Mathematique 348 (2010) 1217-1220. doi:10.1016/j.crma.2010.10.006

[17] E. Burman, P. Hansbo, Fictitious domain finite element methods using cut elements: I. A stabilized Lagrange multiplier method, Computer Methods in Applied Mechanics and Engineering 199 (4144) (2010) 2680 - 2686. doi:10.1016/j.cma. 2010.05 .011

[18] E. Burman, P. Hansbo, Fictitious domain finite element methods using cut elements: II. A stabilized Nitsche method, Appl. Numer. Math 62 (4) (2012) 328 - 341. doi:10.1016/j.apnum.2011.01.008

[19] E. Burman, P. Hansbo, An Interior-penalty-stabilized Lagrange multiplier method for the finite-element solution of elliptic interface problems, IMA J. Numer. Anal. 30 (2010) 870-885. doi:10.1093/imanum/drn081

[20] A. Johansson, M. G. Larson, A high order discontinuous Galerkin Nitsche method for elliptic problems with fictitious boundary, Numerische Mathematik 123 (2013) 607-628. doi:10.1007/s00211-012-0497-1

[21] M. Garzon, N. Bobillo-Ares, J. A. Sethian, Some free boundary problems in potential flow regime using the level set method, in: Fluid Mechanics and Pipe flows: Turbulence, Simulations and Mechanics, Nova Publishers, New York, 2009.

[22] A. Hansbo, P. Hansbo, An unfitted finite element method, based on Nitsche's method, for elliptic interface problems, Comput. Method. Appl. M. 191 (47-48) (2002) 5537 - 5552. doi:10.1016/S0045-7825(02) 00524-8.

[23] A. Hansbo, P. Hansbo, M. G. Larson, A finite element method on composite grids based on Nitsche's method, ESAIMMath. Model. Num. 37 (3) (2003) 495-514. doi:10.1051/m2an:2003039

[24] E. Burman, A Penalty-Free Nonsymmetric Nitsche-Type Method for the Weak Imposition of Boundary Conditions, SIAM J. Numerical Analysis 50 (2012) 1959-1981. doi:10.1137/10081784X.

[25] W. Lorensen, H. Cline, Marching cubes: A high resolution 3D surface construction algorithm, Comput. Graphics 21 (1978) 163-169. doi:10.1145/37401.37422

[26] G. Treece, R. Prager, A. Gee, Regularised marching tetrahedra: improved iso-surface extraction, CUED/F-INFENG/TR 333, Cambridge University Engineering Department (September 1998). doi:10.1016/S0097-8493(99)00076-X

[27] C. Cattani, A. Paoluzzi, Boundary integration over linear polyhedra, Computer-Aided Design 22 (1990) 130 - 135 . doi: 10.1016/0010-4485(90)90007-Y 
[28] M. G. Larson, A. Massing, $\$ \mathrm{~L}^{\wedge} 2 \$$-error estimates for finite element approximations of boundary fluxes, ArXiv eprints arXiv: 1401.6994

[29] S. Balay, et al., PETSc Web page, http://www.mcs.anl.gov/petsc (2014).

[30] S. Osher, J. A. Sethian, Fronts propagating with curvature dependent speed: algorithms based on Hamilton-Jacobi formulations, J. Comput. Phys. 79 (1988) 12-49. doi:10.1016/0021-9991(88)90002-2

[31] D. Adalsteinsson, J. Sethian, Transport and diffusion of material quantities on propagating interfaces via level set methods, Journal of Computational Physics 185 (1) (2003) 271-288. doi:10.1016/S0021-9991(02)00057-8

[32] D. Chopp, Some improvements of the fast marching method, SIAM J. Sci. Comput. 23 (2001) 230-244. doi:10.1137/ S106482750037617X

[33] J. A. Sethian, Level Set Methods and Fast Marching Methods, Cambridge University Press, 1999.

[34] D. Leppinen, J. Lister, Capillary pinch-off in inviscid fluids, Physics of fluids 15 (2003) 568-578. doi:10.1063/1.1537237

[35] A. M. Gañán-Calvo, J. M. Montanero, Revision of capillary cone-jet physics: Electrospray and flow focusing, Phys. Rev. E 79 (2009) 066305. doi:10.1103/PhysRevE.79.066305

[36] S. I. Betel, M. A. Fontelos, U. Kindeln, O. Vantzos, Singularities on charged viscous droplets, Physics of Fluids 18 (5). doi:10.1063/1.2204044

[37] M. A. Fontelos, U. Kindeln, O. Vantzos, Evolution of neutral and charged droplets in an electric field, Physics of Fluids 20 (9). doi:10.1063/1.2980030

[38] C. Ferrera, J. M. Lpez-Herrera, M. A. Herrada, J. M. Montanero, A. J. Acero, Dynamical behavior of electrified pendant drops, Physics of Fluids 25 (1). doi:10.1063/1.4776238

[39] O. Basaran, T. W. Patzek, R. E. Benner, L. E. Scriven, Nonlinear oscillations and breakup of conducting, inviscid drops in an externally applied electric field, Industrial \& Engineering Chemistry Research 34 (10) (1995) $3454-3465$. doi:10.1021/ie00037a034

[40] J. C. Burton, P. Taborek, Simulations of coulombic fission of charged inviscid drops, Phys. Rev. Lett. 106 (2011) 144501. doi:10.1103/PhysRevLett.106.144501

[41] R. T. Collins, K. Sambath, M. T. Harris, O. A. Basaran, Universal scaling laws for the disintegration of electrified drops, Proceedings of the National Academy of Sciences 110 (13) (2013) 4905-4910. doi:10.1073/pnas.1213708110

[42] E. Giglio, B. Gervais, J. Rangama, B. Manil, B. A. Huber, D. Duft, R. Müller, T. Leisner, C. Guet, Shape deformations of surface-charged microdroplets, Phys. Rev. E 77 (2008) 036319. doi:10.1103/PhysRevE.77.036319

[43] R. L. Grimm, J. L. Beauchamp, Dynamics of field-induced droplet ionization: time-resolved studies of distortion, jetting, and progeny formation from charged and neutral methanol droplets exposed to strong electric fields, The Journal of Physical Chemistry B 109 (16) (2005) 8244-8250. doi:10.1021/jp0450540

[44] R. T. Collins, J. J. Jones, M. T. Harris, O. A. Basaran, Electrohydrodynamic tip streaming and emission of charged drops from liquid cones, Nature Phys. 4 (2008) 149-154. doi:10.1038/nphys807. 